\title{
Sur le régime et spécialement sur les variations saisonnières des cours d'eau brésiliens
}

\section{On the regime and particularly on the seasonal variations of the rivers in Brazil}

\author{
PAIR M. PARDE \\ PROFESSEUR A L'UNIVERSTTÉ DE RHENOBHEF \\ ET A L'ÉCOLE NATIONALE SUP'́RIEURE D'ÉLECTHICITE ET D'HYDNALIUUL
}

\begin{abstract}
Explications liminaires. Calle communication n'est qu'une partie, la plus originale, d'une itude beancoup plus vaste, dont lauteur pré sente en raccourci les anfres chapitres: abondance molfenne anmuelle - étiages - crues phenonènes impatables is la dynamique flumiale. Les variations saisomnitres marient selon la lattade des divers rios, et lantem distingus" an régime tropical anstral, un régime tropieal boréal altéré, des régimes équaloriana et sub. équatorianx, enfin des regimes complexes changeants. Pour chacun d'eux, l'auteur analyse les bassins ou les cours d'eau les plus représentalifs dudit régime, et note au passage le comportement insolite et déroutant de certains dentre ces derniers.
\end{abstract}

\begin{abstract}
Preliminary explanations. This paper is anly " part, the most original, of a mush wider study, of which the author briefly antlines the other chapters: mean annual abundance--tow water-floods-phenomena that an be ascribed to riner dipnamics.

The seasonal matations party atording to the latitude of the different rios, and the author distinguishes between an atstrul tropical regime, an altered boreal tropical regime, equatorial and sub-equatorial reqimes, and finally complex changing regimes. For carh of them the athor analyses the most representatine catehment areas or water conrses of the particular regime. and carefully note the unusual and confusing behaninur of some of these regimes.
\end{abstract}

\section{INTRODUCTION}

La Houille Blanche voulant honorer, par un numéro spécial, les aménagements hydrauliques effectués au Brésil, m’a demandé un article d'ensemble sur les rivières qui drainent cet immense territoire, et qui comprennent le plus puissant des fleuves terrestres, à savoir l'Amazonc.

J'ai done élaboré un mémoire intilulé : Quelques aperçus relatifs à l'hydrologie brésilienne.

Ce texte ne pouvait traiter que de manière tris succincte un sujet énorme, el d'ailleurs, insuffisamment connu pour une partie considerarable du réseau fluvial en question, malgré le développement remarquable de l'Hydrométrie (*)

(*) Les donnees hydrométriques principales sont des Annurites flumiometriques, etablis d'après des milliers de jaugeages modernes, mais seulement relatifs à la région $i_{a}$ plus peuplée du pays, e'est-à-dire au sud-est (Bassins du Rio-Grande qui, joint au Paranaiba, constitue le Parana, du Paraïba do Sul, du Sĩo Francisco, du Doce, de l'Uruguay, du Jequirica, du Pardo, du Jequitinhonha, etc.). En outre, un bel ouvrage, en deux volumes, de M.F.A. Solbaxo: Regimen y approvechiamento de la Red fluvial argentina, contient des données sur l'Lrustuay et quelques secteurs brésiliens du Palaguay et du Parana. M. Mafinivos Tornes a publie une belle éfude dans ce pays déjà si riche en réalisations scientifiques et techniques. Cependant mon mémoire s'est révélé trop long pour qu'on l'imprime sans

en deux volumes sur le Paraiba do sul. Pour le résean amazonien, par malheur, nous ne disposons que de données en hauteurs d'eau. Foree nous dtait d'evaluer lias débits moyens annuels du fleuve monstrueux et de ses tributaires d'après les prépitations. Mais les stalions pluviométriques sont très elairsemés en Amazonie Cependant le superbe Atlas plaviometrique du Brisil, publiée en 1948 par la Divisüo de Aguas, du Minislère lederal de l'Agrieuliure $(60 \times 45 \mathrm{~cm}, 49$ pages, 25 figures $)$ nous a foumi sur ce point des renseignements pricieux. Et jai ulilisé avee un graud profit la belle carte au $1 / 5.000 .000^{\circ}$ du Brésit, publiéce en 1950 par l'Instilut Brésilien de Géographie et de Statistique.

Tous ces documents imprimes, et des quaulites de chiffres dactylographies mont ité fournis gêncheuscment par les Directeurs de la Divisao de Aguas, nolamment par M. Antonio José Arytes de Sovzh, puis par son sucesesscur, M. Waldemar José de Canvalho.

J'avais moi-même, précédemment, fait paraitre un article sur les Variations saisonnieres de l'Amazone (Annales de Géograplie, 1936, pp. 502-511) et un autre sur le Régime dés conts d'ean argentins, urughugens, paragurgyens et stu-bresiliens (Estudios frografieos, do Madrid, Ano XII, nov. 1952, [p. 616-648, 6 fig.). 


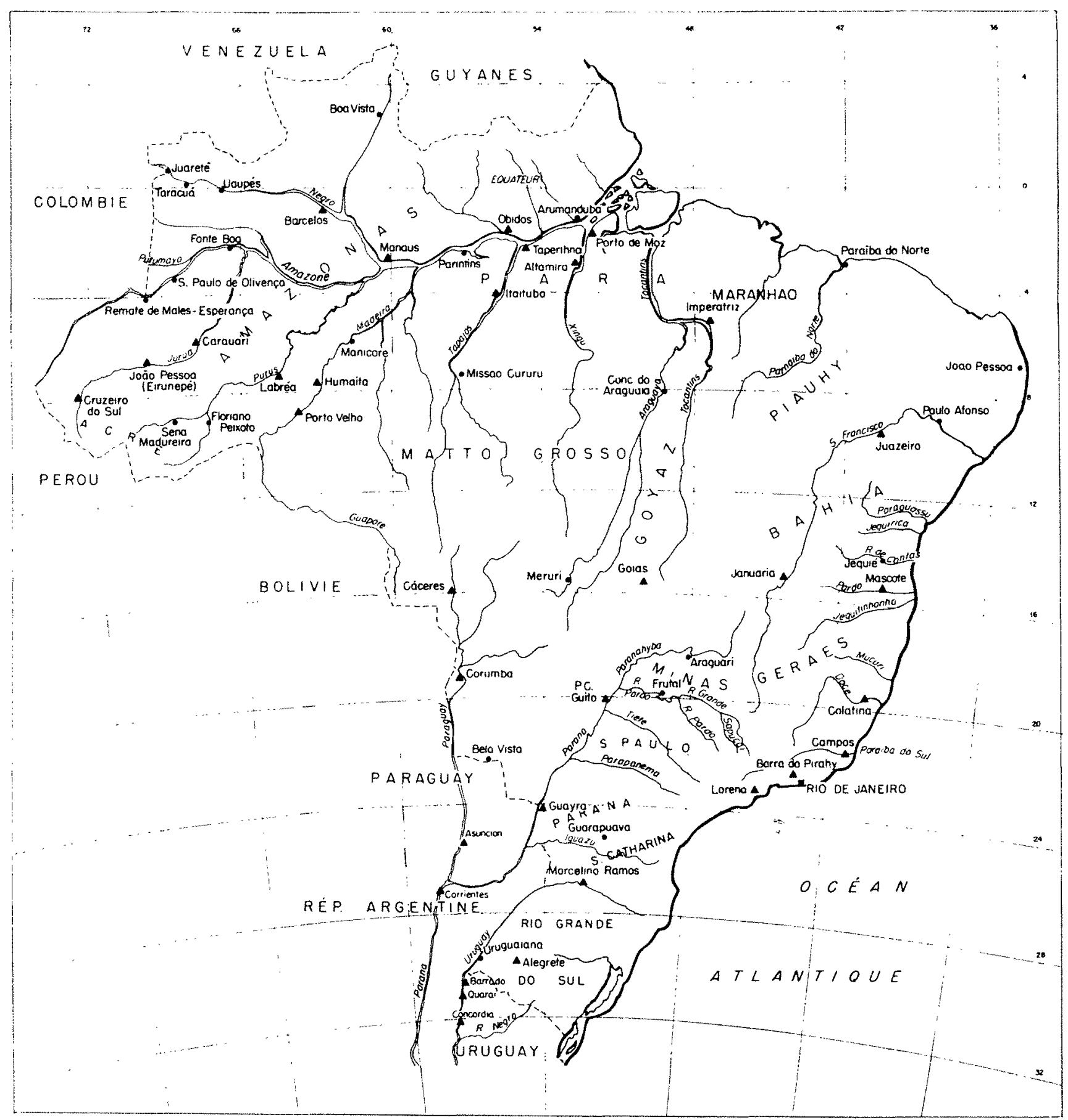

Fir. 1. - Carte du résean fluvial, des principales stations hydrométriques et de quelquess stations pluviométriques ( $y$ compris toutes celles qui étaient en service dans le centre nord et ouest en 1938 .

A stations hydrométriques

- stations pluviométriques

dépasser gravement les limites acceptables dans la publication envisagée. J'aurais donc pu en réduire toutes les parties de moitié ou des deux tiers. Mais la Direction de la Houille Blanche a estimé comme moi que cette contraction d'une étude déjà très abrégée malgré son ampleur, ne pouvait se faire sans dommage pour le meilleur de la substance et pour la clarté de l'analyse. On a donc préféré reproduire le texte intégral d'un seul chapitre, celui qui concerne les variations saisonnières. On a choisi cet élément du régime parce que c'est probablement lui qui présentera le plus d'originalité, en raison de l'hydrologie, ćtrange pour la plupart des lecteurs, qui se des- 
sine dans un secteur assez étendu de la région côtière, de part et d'autre de Bahia. D'autre part, il n'est point exclu que la Honille Blanche fasse imprimer ou polycopier plus tard lensemble du mémoire. Cependant, on résumera pour commencer les chapitres non reproduits ci-dessous.

\section{1. - ELEMENTS HYDROLOGIQUES AUTRES QUE LES VARIATIONS SAISONNIERES}

\section{A) Abondance moyenne annuelle}

L'abondance moyenne annuelle se caractérise par les modules ou débits moyens annucls spécifiques (en lit. sec. par $\mathrm{km}^{2}$ de surface réceptrice ou en millimètres de pluie écoulée), puis par les modules bruts, présentés tels quels sans égard aux superficies drainées. Elle est dominée par les faits suivants (fig. 2):

1" L'abondance pluviale est en général très grande sur la majeure partie du Brésil, qui recoit plus de $1.500 \mathrm{~mm}$ sur des millions de $\mathrm{km} \mathrm{m}^{2}$ et plus de $2.000 \mathrm{~mm}$ sur un tiers peut-être du pays; on recueille même $3.000 \mathrm{~mm}$ et plus dans l'extrême-ouest amazonien, et par plaques isolées et restreintes sur les reliefs sud-orientaux yoisins de l'Océan;

$2^{\circ}$ Cependant, une étendue supérieure à celle de la France, soit plus de $800.000 \mathrm{~km}^{2}$ (un dizieme de la surface totale) dans l'angle oriental du Brésil (bassin du São Francisco et zones voisines) n'encaisse que moins de $1.000 \mathrm{~mm}$, avec des chiffres inférieurs à $500 \mathrm{~mm}$ sur des zones relativement spacieuses;

$3^{\circ} \mathrm{Or}$, sous les températures moyennes annuelles de 18 à $26^{\circ}$ qui règnent au Brésil, l'évapotranspiration, c'est-à-dire le déficit d'écoulement ou différence entre pluies tombées et pluies écoulées, ne peut qu'être considérable, au point de réduire à des valeurs très faibles ou infimes les parts des débits dans la région d'arrosage médiocre ci-dessus localisée. Et on a lieu de croire que dans les secteurs les plus pluvieux, le déficit plafonne à des valeurs comprises entre 1.300 et 1.500 ou $1.600 \mathrm{~mm}$, selon les autres facteurs régionaux.

En conséquence, dans la zone peu pluvieuse orientale, certaines rivieres, semblables en cela aux ouadis maghrébins ou aux creeks nord-américains, débitent moins de 1 et même de $0,5 \mathrm{l} / \mathrm{s}$ par $\mathrm{km}^{2}$, soit de 31,5 et de $15,75 \mathrm{~mm}$. Entre Januaria et Juazeiro, pour $289.228 \mathrm{~km}^{2}$, le São Francisco ne gagne que $2,50 \mathrm{l} / \mathrm{s}$ par $\mathrm{km}^{2}$. Des fleuves côtiers voisins ne roulent que 1,62 l/s par $\mathrm{km}^{2}$ (Rio de Contas), 0,488 (Itapicuru), 2,3 (Rio Pardo) contre 6,75 à 7 pour la Seine à Paris. Le bassin total du São Francisco n'émet sans doute que 5,3 à $5,5 \mathrm{l} / \mathrm{s}$ par $\mathrm{km}^{2}$.

En revanche, une foule de rivières, dans les régions accidentées et très arrosées du sud-est (bas- sins de l'Lruguay, du Parana et de ses affluents, Iguazu, Rio Grande el autres, puis du Paraiba do Sul, derrière Rio de Janeiro, ete.) débitent pour des petites surfaces 25 à $40 \mathrm{l} / \mathrm{s}$ par lim², et pour 10.000 à $50.000 \mathrm{~km}^{2}$ de 15 à $25 \mathrm{l} / \mathrm{s}$ par km² (25) pour l'Iguiza, 18 à 20 pour le Rio Grande). En conséquence, on trouve environ 14 pour les $700.000 \mathrm{~km}^{2}$ du Parana a Guayra, 6,7 seulement pour le Paraguay, à sa sortie du Brésil, mais $1 \overline{3}$ à 17 pour l'Uruguay brésilien.

Pour le systeme géant de l'imazone, on est réduit à ne raisonner que d'après les valeurs approximatives des pluies et des déficits d'écoulement probables. On trouve de la sorte des chiffres relativement formidables étant donné les ctendues des bassins considerés, par exemple quelque $30 \mathrm{l} / \mathrm{s}$ par $\mathrm{km}^{2}$ (comme pour l'Isere, l' $\mathrm{hm}$, ou le Rhin à Bâle) pour $300.000 \mathrm{~km}^{2}$ du Purus (*) et $200.000 \mathrm{~km}$ " du Jurua, 35 a 40 pour 150.000 à $175.000 \mathrm{~km}^{2}$ du Yapura, 1 t ou 15 pour 700.000 à $800.000 \mathrm{~km}^{2}$ du Tocantins, 15 à 17 pour $500.000 \mathrm{~km}^{2}$ du Xingsu, 16 a 18 pour 500.0010 $\mathrm{km}^{2} \mathrm{du}$ Tapajoz, et pour 1.000 .000 de $\mathrm{km}^{2} \mathrm{de}$ la Madeira, 20 à 22 pour $500.000 \mathrm{~km}^{2}$ du Rio Negro; et choses lout a fait fantastiques, of sans aulre exemple dans le reste du globe, 23 à 25 pour $3.300 .000 \mathrm{~km}^{2}$ de l'Amazone au confluent avec le Rio Negro inclus, puis 18 à $20 \mathrm{l} / \mathrm{s}$ par $\mathrm{km}^{2}$ sinon plus pour les $5.600 .000 \mathrm{~km}^{2}$ de tout le bassin amazonien au confluent du Xingu inclusivement.

Cela ferait en gros 100.000 a $110.000 \mathrm{~m} \% / \mathrm{s} \mathrm{con-}$

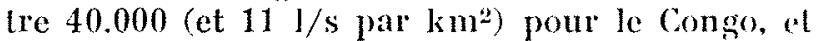
$30.000(17,6)$ pour le Yang-tsé-Kiang. On aurail 16.000 à $18.000 \mathrm{~m}^{3}$ pour la Madeira, 10.000 it 12.000 pour le Rio Negro, ou pour le Tocantins, 8.0000 at 10.000 pour le Purus, et pour le Xingu, comme pour le Tapajoz. D'après des chifres moins sujets a caution, on peut admettre $\$ .000$ a $10.000 \mathrm{HI}^{3}$ pour le Parana aux chutes de Guayra, 10.500 a 11.500 pour ce fletive à Posadas, en Argentine, et 15.000 à 16.000 dans le même pays à Corrientes, grâce à l'appoint du Paraguay $\$ 4.500 \mathrm{~m}^{3}$, dont $3.000 \mathrm{~m}^{3}$ venant du Brésil). L'Uruguay donne 4.000 at $4.500 \mathrm{~m}^{3}$ à son embouchure, dont 3.250 a 3.500 originaires du Brésil.

Le São Francisco apporte a la mer quelque $3.300 \mathrm{~m}^{3}$, le Paraïba do Sul et le Doce, 1.000 , le Rio Grande et lc Paranaïba, chacun 2.250 à 2.500 .

(*) Les surfaces réceptrices données pour ces grandes rivières ne sont que très grossierement approximatives. 


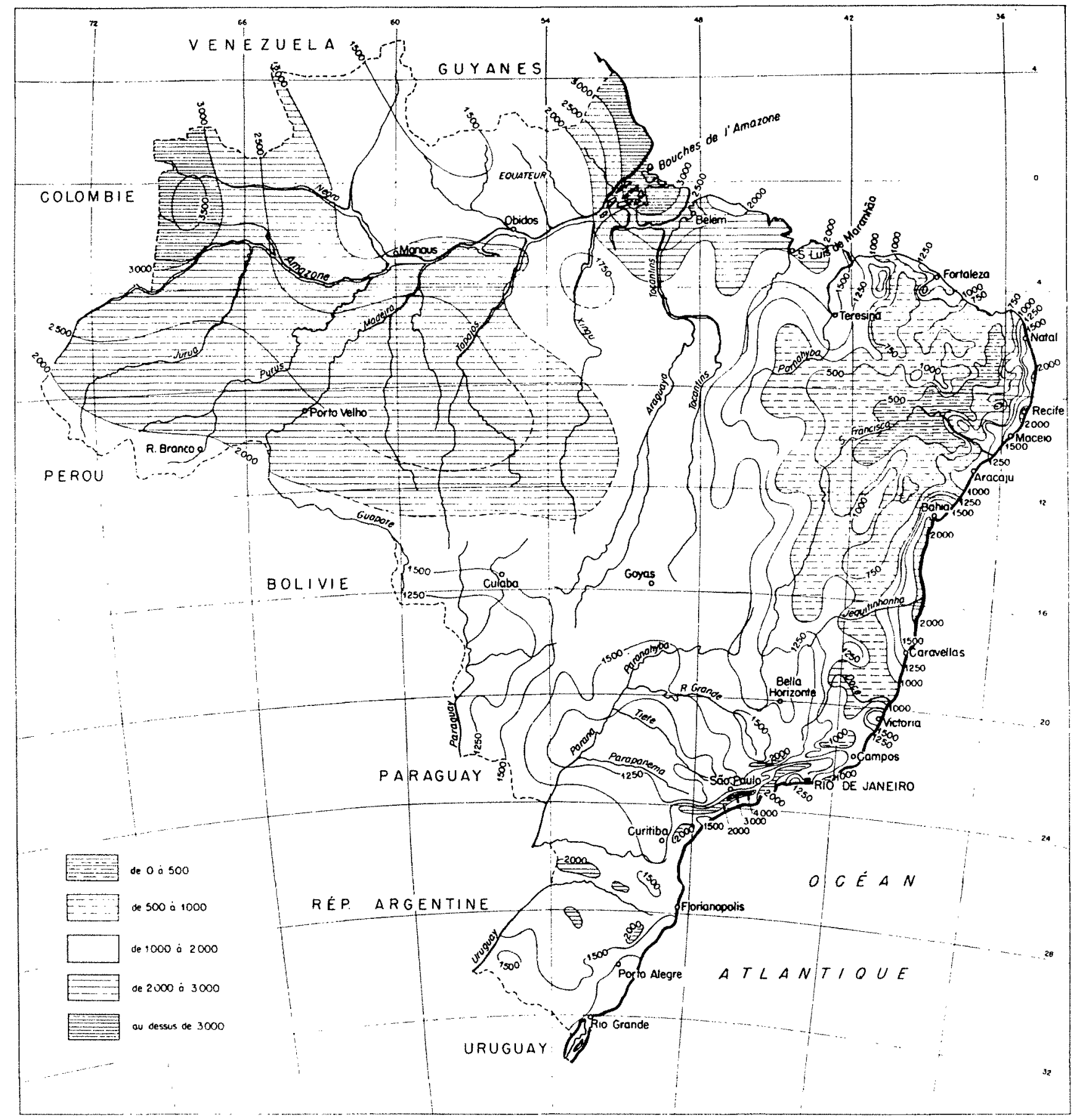

Fu. 2. - Carte des pluies. - Isohyètes annuels.

\section{B) Etiages}

Un des faits les plus importants de l'hydrologie brésilienne est l'abondance conservée par les ètiages de nombreuses rivières; et d'abord, dans toute la zone sud-orientale ì climat tropical typique, avec grandes pluies d'été, médiocrité pluviale ou sécheresse en hiver. Le fait doit d'ailleurs tenir non à ces répartitions pluviales, mais à des pouvoirs de rétention remarquahles possédés par des terrains. Il s'agit de roches en place basaltiques ou plus communément gréseuses, et surtout, semble-t-il, d'épais manteaux superficiels de décomposition fournis aux dépens de ces roches ou des éléments cristallins imperméables à l'état de masses non fissurées, qui abondent.

Quoi qu'il en soit, le minimum minimorum relativement catastrophique du l'arana à Posadas, en 1944, représentait encore $3.200 \mathrm{~m}^{3}$, soit 4 l/s par $\mathrm{km}^{2}$ (4,3 pour le Rhône inférieur, moins de 1 pour le Mississipi, de 0,5 pour la Seine). Et dans les réseaux de ce fleuve ou du Paraïba méri- 
dional, ou du haut São Francisco, à l'issue de surfaces modérées ou restreintes (quelques centaines de $\mathrm{km}^{2}$ ), les étiages minima connus sont encore généralement compris entre 4 et 8 ou $10 \mathrm{l} / \mathrm{s}$ par $\mathrm{km}$, valeurs dignes des chiffres trouvés pour les rivières nivales des Alpes françaises ou suisses, el pour les cours d'eau pyrénéens septentrionaux.

Puis, sans avoir la-dessus de certitude, nous avons lieu de supposer que des chiffres aussi avantageux, ou cncore plus imposants, pour des causes géologiques, et en raison de la longue durée des pluies qui garnissent les réserves souterraines, caractérisent une quantité, peut-être la majeure partie des rivières amazoniennes à l'issue de petites surfaces. Sur les troncons principaux de ce bassin, les minima les plus faibles doivent encore ètre honorables (quelques $1 / \mathrm{s}$ par $\mathrm{km}^{2}$ ) on magnifiques. Sur l'Amazone lui-même, ces influences sont encore renforcées par les compensations interrégionales que nous verrons jouer dans les variations saisonnières. Et nous ne serions point surpris que des débits déjà rares par leur faiblesse relative atteignent encore 7 a $8 \mathrm{~J} / \mathrm{s} \mathrm{par} \mathrm{km}^{2}$ à Obidos, ou à la fin du cours; soit respectivement 31.000 a 36.000 , puis 39.000 a $45.000 \mathrm{~m}^{3}$, c'est-ì-dire aulant, en pénurie, que le module du Congo.

Par contre, à l'extrême sud-est, un climat moins régulier dans ses vicissitudes, et surtout, selon la vraisemblance, des terrains à faible pouvoir de rétention dépriment les écoulements en étiage. L'Uruguay inférieur à Concordia, hors du Brésil, est tombé en mai 1935 à $250 \mathrm{~m}^{3}$, soil a $1 \mathrm{l} / \mathrm{s}$ par $\mathrm{km}^{2}$. A l'amont, certains de ses tributaires s'anémient plus encore, comme la plupart des rivières pluviales européennes.

\section{C) Les crues}

En opposition (mais aussi en corrélation) avec ces indigences, le bassin de l'Uruguay semble éprouver des crues très puissantes. Ce fleuve aurait roulé $30.000 \mathrm{~m}^{3}$ pour $227.000 \mathrm{~km}^{2}$ a Federacion, le 12 mai 1941 , maximum comparable à ceux du très dangereux Ohio. Plus alu nord, l'Iguazu aurail débité le 9 juin 1936, au lieu de ses grandes chutes, 25.000 m", soil, pour 48.000 $\mathrm{km}^{2}$, plus de $500 \mathrm{~J} / \mathrm{s}$ par $\mathrm{km}^{2}$, chiffre relativement formidable. Et tout à fait fantastique encore aurait élé le déluge du Jacui en mai 1941 : $40.000 \mathrm{~m}^{3} / \mathrm{s}$ pour moins de $60.000 \mathrm{~km}^{2}$. Dans celte zone, les inondations graves ont lieu surtoul au printemps ef en aulomne.

Les crues séricuses se produisent presque exclusivement en saison chaude el surtout en ité (janvier-mars) dans toute la vaste zone du climat tropical typique, par exemple pour le Paraguay supérieur, le réseau du Parana en amont de l'lguazu, le Paraỉa do Sul, le São Francisco, ete., ou sur les cours supérieurs des affuents amazoniens de droite; puis, avec un retard atleignant quelques semaines à un mois, sur les tronçons inférieurs des mèmes rivières, pour les causes que l'on verra en étudiant les variations saisonnieres. Au nord du bassin, la fréquence est la plus marquáe en mai-juillet. Il semble que dans presque toute cetle valste zone, les débits maxima spéciliques à l'issue de surfaces dépassant quelques milliers de lim², soient bien moins massifs que pour l'Uruguay, l'Iguazu, le Jacui peut-être, et leurs tributaires. Celte infériorilí sensible me parail en lout cas guere douteuse pour le Paranal, qui aurait débité cependant en juin 1905 (exception saisonnière) $30.000 \mathrm{~m}^{3}$ ou plus pour $700.000 \mathrm{~km}^{2}$ ì Guayra, 35.000 a 40.000 à Posadas (peut-ètre 50.000 ou plus à Corrientes) loin au-delà du Brésil.

Les crues, elles aussi estivales en principe, du Paraguay supérieur sont, alu cours de leur propagation dans le cours moven, retardées de plusieurs mois of aphaties par l'étalement sur l'immense champ d'inondation du Pantunul. Grâce à ce phénomene, on ne comnailrail pas plus de 6.500 $\mathrm{m}^{3}$ a Puerto-Sitstre, soit $15 \mathrm{l} / \mathrm{s}$ sentement par km². A Juazeiro, le Sia Francisco a roule $14.400 \mathrm{~m}^{3}$ en 1949 .

Les débits maxima de lidmazone ont presque tous lieu en mai et juin sur le cours inférieur. D'après les modules, il nous semble extrèmement probable qu'ils peuvent depasser $150.00\left(0 \mathrm{~m}^{3}\right.$ à Obidos, el 160.000 à Santarem, chiffres inconnus à beaucoup près, ailleurs dans le monde. EI peut-ètre les maxima exceptionnels frisent-ils $200.000 \mathrm{~m}^{3}$. Une grande crue a d'ailleurs lien tous les ans sur le bas Amazone, régulariti encore plus sensible que sur les rivieres tropicales a petits bassins, el qui contraste avec le desordre relevé à ce point de vue sur l'Urugualy el ses tributaires.

Enfin, des crues très brutales et peut-itre exorbitantes pour leurs débits spicifiques se bealisent sur des surfaces restreintes: quelques dizaines à quelques milliers de km². Le Professeur H. O. Reingy-STEminesg en cite une de ce gente, survenue le 15 décembre 1948, et qui dévasta $1.500 \mathrm{~km}^{2}$, drainés par quelques petits affluents du Paraiba do Sul. Mais rien ne nous révile en quelles zones peuvent se produire ces phenomines, ni quels chiffres pluviaux ou hyolrométriques expriment leur violence. A tort ou a raison, nous inclinons à les croire moins formidables que les crues méditerranéennes d'surope, par exemple, ou que les « flash floods z encore plus terribles des Etats-Tnis.

\section{D) Faits de dynamique fluviale.}

Pour bien caractériser les jiviores bresiliennes, il laudrait pouvoir déborder des cadres fixés par 


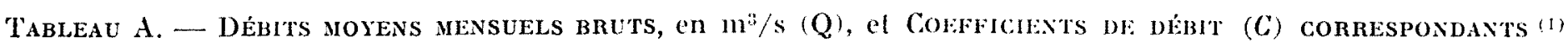

\begin{tabular}{|c|c|c|c|c|c|c|c|c|c|c|c|c|c|c|c|c|c|c|}
\hline RIVIĖRE & STATION & $\begin{array}{l}\text { Périodes } \\
\text { d'obser- } \\
\text { vations }\end{array}$ & $\begin{array}{c}\text { Surface } \\
\text { réceptrice } \\
\mathrm{km}^{2}\end{array}$ & & Janv. & Fév. & Mars & Avril & Mai & Juin & Juil. & Åoût & Sept. & Oct. & Nov. & Déc. & $\begin{array}{l}\text { Moy. } \\
\text { Ann. }\end{array}$ & $q^{(2)}$ \\
\hline Ibirapuita. & Alegrete... & $1941-1949$ & 5,850 & $\begin{array}{l}Q \\
C\end{array}$ & $\begin{array}{c}48 \\
0,549\end{array}$ & $\begin{array}{l}21 \\
0,24\end{array}$ & $\begin{array}{c}19,7 \\
0,225\end{array}$ & $\begin{array}{c}98,5 \\
1,127\end{array}$ & $\begin{array}{l}173 \\
1,978\end{array}$ & $\begin{array}{r}101,5 \\
1,161\end{array}$ & $\begin{array}{l}107 \\
1.223\end{array}$ & $\begin{array}{l}101 \\
1,155\end{array}$ & $\begin{array}{c}91,3 \\
1,043\end{array}$ & $\begin{array}{c}174 \\
1,990\end{array}$ & $\begin{array}{l}61 \\
0,697\end{array}$ & $\begin{array}{c}54 \\
0,617\end{array}$ & 87,5 & 15 \\
\hline Paraiba do Sul & Cacapava. & || $923-1942 \mid$ & 8.490 & $\underset{C}{Q}$ & $\begin{array}{r}222,8 \\
1,488\end{array}$ & $\begin{array}{r}249.3 \\
1.664\end{array}$ & $\begin{array}{l}239 \\
1,595\end{array}$ & $\begin{array}{r}178,6 \\
1,192\end{array}$ & $\begin{array}{r}131,8 \\
0,878\end{array}$ & $\begin{array}{l}112 \\
0.747\end{array}$ & $\begin{array}{c}93,5 \\
0,625\end{array}$ & $\begin{array}{c}84,8 \\
0,566\end{array}$ & $\begin{array}{l}94,8 \\
0,633\end{array}$ & $\begin{array}{r}108,7 \\
0,725\end{array}$ & $\begin{array}{r}113,5 \\
0,755\end{array}$ & $\begin{array}{l}168 \\
1,122\end{array}$ & 150 & 17,7 \\
\hline$-s^{\circ}-$ & Barra do Pirai.. & $1922-1942$ & 16.350 & $\underset{C}{Q}$ & $\begin{array}{r}507 \\
1,53\end{array}$ & $\begin{array}{r}573 \\
1,73\end{array}$ & $\begin{array}{c}560 \\
1,69\end{array}$ & $\begin{array}{l}404 \\
1,22\end{array}$ & $\begin{array}{r}283 \\
0,85\end{array}$ & $\begin{array}{l}239 \\
0.72\end{array}$ & $\begin{array}{l}197 \\
0,59\end{array}$ & $\begin{array}{r}161,5 \\
0.49\end{array}$ & $\begin{array}{c}187 \\
0,56\end{array}$ & $\begin{array}{l}2.12 \\
0,64\end{array}$ & $\begin{array}{c}256 \\
0.78\end{array}$ & $\begin{array}{l}396 \\
1,20\end{array}$ & 331,3 & 20,3 \\
\hline$-\dot{a}^{0}-$ & Campos.. & $1928-1933$ & 56.322 & $\begin{array}{l}Q \\
C\end{array}$ & $\begin{array}{r}1.554 \\
1,63\end{array}$ & $\begin{array}{r}1.639 \\
1.715\end{array}$ & $\begin{array}{r}1.443 \\
1,52\end{array}$ & $\begin{array}{r}1.048 \\
1,095\end{array}$ & $\begin{array}{r}783 \\
0,82\end{array}$ & $\begin{array}{r}713 \\
0.745\end{array}$ & $\begin{array}{l}598 \\
0,626\end{array}$ & $\begin{array}{l}546 \\
0,571\end{array}$ & $\begin{array}{l}519 \\
0.543\end{array}$ & $\begin{array}{l}587 \\
0,615\end{array}$ & $\begin{array}{c}770 \\
0,805\end{array}$ & $\begin{array}{l}1.275 \\
1,305\end{array}$ & 956 & 17 \\
\hline Sao Francisco. & Porto Réal. . & $1928-1942$ & 4.836 & $\stackrel{Q}{C}$ & $\begin{array}{r}181,4 \\
1,90\end{array}$ & \begin{tabular}{|c|}
185,3 \\
1.94
\end{tabular} & $\begin{array}{c}156 \\
1,632\end{array}$ & $\begin{array}{c}104,4 \\
1,092\end{array}$ & $\begin{array}{l}70,9 \\
0.742\end{array}$ & $\begin{array}{c}53.7 \\
0.562\end{array}$ & $\begin{array}{c}45,7 \\
0,478\end{array}$ & $\begin{array}{c}35.7 \\
0.374\end{array}$ & $\begin{array}{c}33,7 \\
0,352\end{array}$ & $\begin{array}{c}38,2 \\
0,398\end{array}$ & $\begin{array}{c}76,2 \\
0,795\end{array}$ & $\begin{array}{c}165,8 \\
1,734\end{array}$ & 95,6 & 19,8 \\
\hline$-d^{0}-$ & Juazeiro. & $1929-1942$ & 490.769 & $\underset{C}{Q}$ & $\begin{array}{r}4.725 \\
1,752\end{array}$ & $\begin{array}{r}4.784 \\
1.777\end{array}$ & $\begin{array}{c}4.702 \\
1,75\end{array}$ & $\begin{array}{r}3.675 \\
1,365\end{array}$ & $0,3.498$ & $\begin{array}{c}1.692 \\
0,628\end{array}$ & $\begin{array}{r}1.438 \\
0,534\end{array}$ & $\left.\right|_{0,463} ^{1.248}$ & $\begin{array}{r}1.094 \\
0.406\end{array}$ & $\mid \begin{array}{l}1.225 \\
0.459\end{array}$ & $\begin{array}{l}1.882 \\
0,699\end{array}$ & $\begin{array}{r}3.362 \\
1.249\end{array}$ & 2.694 & 5,48 \\
\hline Rio Grande. & Bon Jardim.... & $1939-1945$ & 486 & $\stackrel{Q}{C}$ & $\begin{array}{c}23,2 \\
1,616\end{array}$ & \begin{tabular}{c|}
25.3 \\
1.770
\end{tabular} & \begin{tabular}{|c|}
22,5 \\
1,563
\end{tabular} & $\begin{array}{c}15,9 \\
1,111\end{array}$ & $\begin{array}{l}11,4 \\
0,797\end{array}$ & $\begin{array}{l}9,6 \\
0,672\end{array}$ & \begin{tabular}{l|}
8,5 \\
0,594
\end{tabular} & $\begin{array}{l}7 \\
0,489\end{array}$ & $\begin{array}{l}7,3 \\
0,510\end{array}$ & $\begin{array}{l}8,8 \\
0,615\end{array}$ & $\begin{array}{l}12,7 \\
0,888\end{array}$ & $\begin{array}{c}19,5 \\
1,362\end{array}$ & 14,3 & 29,4 \\
\hline$-d^{\circ}-$ & Porto Capetinga. & $1939-1945$ & 25.520 & $\stackrel{Q}{C}$ & $\begin{array}{r}892 \\
1,844\end{array}$ & $\begin{array}{r}918 \\
1.900\end{array}$ & $\begin{array}{c}780 \\
1.614\end{array}$ & $\begin{array}{l}512 \\
1,060\end{array}$ & $\begin{array}{l}363 \\
0.752\end{array}$ & $\begin{array}{l}303 \\
0,627\end{array}$ & $\begin{array}{l}262 \\
0,542\end{array}$ & $\begin{array}{l}214 \\
0,443\end{array}$ & $\begin{array}{c}212 \\
0.439\end{array}$ & $\begin{array}{r}254 \\
0,526\end{array}$ & $\begin{array}{l}381 \\
0,789\end{array}$ & $\begin{array}{c}705 \\
1,459\end{array}$ & 483 & 18,5 \\
\hline$-d^{\circ}-$ & San José de Barra & $1939-1945$ & 52.110 & $\stackrel{Q}{C}$ & 1.620 & $\begin{array}{r}1.720 \\
1.899\end{array}$ & $\begin{array}{r}1.510 \\
1.666\end{array}$ & $\begin{array}{l}991 \\
1,093\end{array}$ & $\begin{array}{l}670 \\
0,740\end{array}$ & $\begin{array}{l}583 \\
0,644\end{array}$ & $\begin{array}{l}510 \\
0,563\end{array}$ & $\begin{array}{l}412 \\
0,455\end{array}$ & $\begin{array}{l}417 \\
0,460\end{array}$ & $\begin{array}{l}501 \\
0,553\end{array}$ & $\begin{array}{c}725 \\
0,800\end{array}$ & $\begin{array}{c}1.216 \\
1,341\end{array}$ & 906,2 & 17,4 \\
\hline Soxpucai... & Porto Carrito.... & $1939-1945$ & $24.062^{\prime}$ & $\stackrel{Q}{C}$ & $\begin{array}{l}648 \\
1,637\end{array}$ & $\begin{array}{r}790 \\
1,996\end{array}$ & $\begin{array}{l}690 \\
1,742\end{array}$ & $\begin{array}{r}427 \\
1.079\end{array}$ & $\begin{array}{l}304 \\
0,769\end{array}$ & $\begin{array}{r}260 \\
0,656\end{array}$ & $\begin{array}{l}230 \\
0,582\end{array}$ & $\begin{array}{r}184 \\
0,465\end{array}$ & $\begin{array}{r}193 \\
0,488\end{array}$ & $\begin{array}{c}223 \\
0.564\end{array}$ & $\begin{array}{c}325 \\
0,822\end{array}$ & $\begin{array}{c}477 \\
1,204\end{array}$ & 396 & 16.4 \\
\hline Igucazu. .... & Porto Uniao. & $1931-1933$ & 23.680 & $\stackrel{Q}{C}$ & $\begin{array}{r}386 \\
0,735\end{array}$ & $\begin{array}{c}392 \\
0,745\end{array}$ & $\begin{array}{r}340 \\
0,645\end{array}$ & $\begin{array}{c}689 \\
1,32\end{array}$ & $\begin{array}{r}873 \\
1,66\end{array}$ & $\begin{array}{l}877 \\
1,665\end{array}$ & $\begin{array}{l}556 \\
1,06\end{array}$ & $\begin{array}{c}232 \\
0,44\end{array}$ & $\begin{array}{l}601 \\
1,14\end{array}$ & $\begin{array}{c}585 \\
1,11\end{array}$ & $\begin{array}{r}314 \\
0,595\end{array}$ & $\begin{array}{c}472 \\
0,90\end{array}$ & 526 & 22,2 \\
\hline Parma & $\begin{array}{l}\text { Corrientes (3). } \\
\text { Argentine)..... }\end{array}$ & $1901-1950$ & 1.936 .000 & $\stackrel{Q}{C}$ & $\begin{array}{r}16.550 \\
\quad 1.015\end{array}$ & $\begin{array}{c}20.400 \\
1.25\end{array}$ & $\begin{array}{r}20.800 \\
1,275\end{array}$ & $\begin{array}{c}19.050 \\
1.17\end{array}$ & $\begin{array}{r}16.900 \\
1,038\end{array}$ & $\begin{array}{c}17.0001 \\
1,043^{1}\end{array}$ & $\begin{array}{l}15.550 \\
0.955\end{array}$ & $\begin{array}{r}12.5001 \\
0.766\end{array}$ & $\begin{array}{r}12.450 \\
0.764\end{array}$ & $\begin{array}{r}14.250 \\
0.874\end{array}$ & $\begin{array}{l}14.700 \\
0,902\end{array}$ & $\begin{array}{r}15.350 \\
0,941\end{array}$ & 16.300 & 8,4 \\
\hline Jequitinhonha. & Itamarati. & 1935-1943 & 61.907 & $\stackrel{Q}{C}$ & $\begin{array}{r}1.247 \\
2,457\end{array}$ & $\begin{array}{r}930 \\
1.832\end{array}$ & $\begin{array}{c}882 \\
1,738\end{array}$ & $\begin{array}{l}421 \\
0,83\end{array}$ & 211 & $\begin{array}{c}162,5 \\
0,321\end{array}$ & $\begin{array}{r}136 \\
0,258\end{array}$ & $\begin{array}{l}118 \\
0,233\end{array}$ & $\begin{array}{l}113 \\
0,223\end{array}$ & $\begin{array}{l}157 \\
0,309\end{array}$ & $\begin{array}{c}652.5 \\
1.286\end{array}$ & 2.064 & 507,5 & 8,2 \\
\hline Doce. & Solatina. & $\mid \begin{array}{r}|938-1944| \\
-1942\end{array}$ & 77.407 & 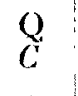 & 1.857 & $\begin{array}{r}1.402 \\
1,44\end{array}$ & 1.436 & $\begin{array}{c}1.060 \\
1.089\end{array}$ & $\begin{array}{l}771 \\
0.792\end{array}$ & $\begin{array}{r}652 \\
0,669\end{array}$ & $\begin{array}{c}584 \\
0,60\end{array}$ & $\begin{array}{l}515 \\
0,528\end{array}$ & $\begin{array}{c}464,5 \\
0,477\end{array}$ & $\begin{array}{l}569 \\
0,584\end{array}$ & $\begin{array}{r}843 \\
0,865\end{array}$ & $\begin{array}{c}1.538 \\
1,580\end{array}$ & 974 & 12,6 \\
\hline Rio Mucuri... & Mairinque & $1938-1943^{\circ}$ & 13.820 & $\stackrel{Q}{C}$ & $\begin{array}{r}188,6 \\
2,22\end{array}$ & $\begin{array}{c}98,5 \\
1,16\end{array}$ & $\begin{array}{r}111,3 \\
1,32\end{array}$ & $\begin{array}{l}82,9 \\
0,975\end{array}$ & $\begin{array}{c}50,2 \\
0,59\end{array}$ & $\begin{array}{r}49,5 \\
0,582\end{array}$ & $\begin{array}{l}39 \\
0.458\end{array}$ & $\begin{array}{l}34 \\
0,400\end{array}$ & $\begin{array}{c}30,8 \\
0.362\end{array}$ & $\begin{array}{c}44,5 \\
0,524\end{array}$ & $\begin{array}{c}82,2 \\
0,967\end{array}$ & \begin{tabular}{|r|}
207,4 \\
2,44
\end{tabular} & 85 & 6,14 \\
\hline Rio de Contas.. & Itapira. & $\mid 936-1943$ & 52.227 & $\stackrel{Q}{C}$ & $\begin{array}{c}67 \\
0,792\end{array}$ & $\begin{array}{l}97,5 \\
1,152\end{array}$ & $\begin{array}{r}131.5 \\
1.554\end{array}$ & $\begin{array}{r}104,4 \\
1,233\end{array}$ & $\begin{array}{r}104,8 \\
1,238\end{array}$ & $\begin{array}{c}78,9 \\
0,932\end{array}$ & $\begin{array}{c}60,2 \\
0,71\end{array}$ & $\begin{array}{r}57,9 \\
0,684\end{array}$ & $\begin{array}{c}41,6 \\
0,492\end{array}$ & $\begin{array}{c}46,6 \\
0,550\end{array}$ & $\begin{array}{c}97,2 \\
1.148\end{array}$ & $\begin{array}{r}128.2 \\
1,514\end{array}$ & 84,7 & 1,62 \\
\hline Cachoeira. . & Itabuna. . & 1936-1943: & 4.078 & $\stackrel{Q}{C}$ & $\begin{array}{c}26,2 \\
0,983\end{array}$ & $\begin{array}{l}29,7 \\
1,117\end{array}$ & $\begin{array}{r}47,3 \\
1,782\end{array}$ & $\begin{array}{c}50,9 \\
1,920\end{array}$ & $\begin{array}{l}34,2 \\
1,285\end{array}$ & $\begin{array}{r}25,7 \\
0,968\end{array}$ & $\begin{array}{l}11,2 \\
0,417\end{array}$ & $\begin{array}{l}8,5 \\
0,319\end{array}$ & $\begin{array}{l}7.6 \\
0,287\end{array}$ & $\begin{array}{l}8,75 \\
0,330\end{array}$ & $\begin{array}{c}48.5 \\
1.828\end{array}$ & $\begin{array}{c}20,4 \\
0,767\end{array}$ & 26,55 & 6,52 \\
\hline Rio Pardo.... & Mascote. & $1937-1943$ & 27.915 & $\stackrel{\mathrm{O}}{\mathrm{C}}$ & $\begin{array}{l}64,3 \\
1,000\end{array}$ & $\begin{array}{c}75,9 \\
1,182\end{array}$ & $\begin{array}{r}100.6 \\
1.567\end{array}$ & $\begin{array}{l}94,8 \\
1,477\end{array}$ & $\begin{array}{c}60,4 \\
0,942\end{array}$ & $\begin{array}{c}50,4 \\
0,785\end{array}$ & $\begin{array}{c}39,6 \\
0.617\end{array}$ & $\begin{array}{l}35.2 \\
0,548\end{array}$ & $\begin{array}{c}30,8 \\
0,480\end{array}$ & $\begin{array}{c}34,2 \\
0,532\end{array}$ & $\begin{array}{c}66,5 \\
1,036\end{array}$ & $\begin{array}{c}117,3 \\
1,829\end{array}$ & 64,2 & 2,3 \\
\hline Jequirica. & Pimenteira Lage. & $\mid 932-1943$ & 5.000 & $\stackrel{\mathrm{Q}}{\mathrm{C}}$ & $\begin{array}{l}8,54 \\
0,706\end{array}$ & $\begin{array}{l}8,87 \\
0,733\end{array}$ & $\begin{array}{c}14.82 \\
1,225\end{array}$ & $\begin{array}{c}10,69 \\
0,884\end{array}$ & $\begin{array}{c}11,36 \\
0,939\end{array}$ & $\begin{array}{c:}12,2 \\
1,02\end{array}$ & $\begin{array}{r}18,25 \\
1,52\end{array}$ & $\begin{array}{c}14,13 \\
1,169\end{array}$ & $\begin{array}{c}11,36 \\
0,938\end{array}$ & $\begin{array}{c}10,5 \\
0,868\end{array}$ & $\begin{array}{l}11,6 \\
0,958\end{array}$ & $\begin{array}{l}12.8 \\
1,058\end{array}$ & 12,1 & 2,42 \\
\hline Itapicuru. & Cajueiro.. & $1935-1943$ & 35.123 & $Q$ & $\begin{array}{l}7,9 \\
0,46\end{array}$ & $\begin{array}{c}14,68 \\
0,856\end{array}$ & $\begin{array}{c}50,2 \\
2,93\end{array}$ & $\begin{array}{c}20,4 \\
1,19\end{array}$ & $\begin{array}{c}26,2 \\
1,528\end{array}$ & $\begin{array}{c}18,3 \\
1,068\end{array}$ & $\begin{array}{c}16,8 \\
0,98\end{array}$ & $\begin{array}{c}16,54 \\
0,965\end{array}$ & $\begin{array}{c}12,23 \\
0,714\end{array}$ & $\begin{array}{l}6,18 \\
0,360\end{array}$ & $\begin{array}{l}6,8 \\
0,397\end{array}$ & $\begin{array}{l}9,46 \\
0,552\end{array}$ & 17,14 & 0,488 \\
\hline & & & & & & & & & & & & & & & & & & \\
\hline
\end{tabular}


l'Hydrologie pure (ou simple élude des régimes) et signaler puis expliquer force phénomènes qui ressortent essentiellement de la Dynamique fluviale : vitesses, puissances tractrices et érosives des courants, profils en long et en travers, transports solides.

Sur ces derniers nous savons très peu de chose. Cependant il paraitt probable que le Parana brésilien charrie relativement très peu de boues: probablement moins de 100 , et plutòt de 50 tonnes par $\mathrm{km}^{2}$ et par an $(500$ à 1.000 pour maintes rivières des grandes Alpes, 2.500 pour le Fleuve Jaune). La cause serait la rareté des roches très délitables en éléments fins, et encore plus la richesse de la couverture végétale, surtout forestì̀re. Mais ce même facteur devrait réduire à des valeurs modérées ou petites la turbidité de l'Amazone qui draine la plus grande sylve équatoriale du monde, dans un bassin extrêmement plat sur la moitié de son étendue, et peu accidenté sur les quatre cinquièmes du reste. $\mathrm{Or}$, le fleuve géant charrierait en moyenne au moins 750 grammes par $\mathrm{m}^{3}$, et peut-être 1.000 , soit 2 ou 2,5 milliards de tonnes, ou encore 400 à 500 tonnes ou plus par $\mathrm{km}^{2}$ et par an; chiffres tout à fait inattendus et relativement énormes (moins de 50 tonnes par $\mathrm{km}^{2}$ et par an pour le Congo!). Si ces valeurs ne sont point fausses, nous estimons qu'on peut les attribuer à l'érosion venue de la partic andine extrême-orientale dụ bassin, ou à la zonc semi-steppique, done mal protégée par la végétation de la haute Madeira au sud-ouest, ou aux érosions latérales exercées dans les méandres remarquables du Purus, du Jurua et d'autres rivières, ou à tous ces facteurs combinés.

Et il faudrait un copieux chapitre pour décrire une autre caractéristique de l'Hydrographie fluviale brésilienne, à savoir le grand nombre des ruptures de pente, rapides ou cataractes, phénomènes qui évoquent les traits analogues des profils en long africains pour les mêmes lalitudes. On citera seulement ici la cataracte grandiose de Paulo Afonso sur le bas Sào Francisco, celle du bas Iguazu ( $80 \mathrm{~m}$ pour l'une et l'autre), puis, sur le Parana, celle de Guayra $(40 \mathrm{~m})$, particulièrement intéressante en raison des débits puissants que l'on a signalés, el qui parfois nivelemaient presque la chute. Ces accidents des profils sonl on ne peut plus favorables aux aménagements hydro-électriques, mais néfastes comme hien l'on pense a la navigation intérieure. Celle-ci peut cependant triompher, avec de heaux tirants d'eau, sur des milliers de kilomètres dans le réseau amazonien.

Et nous en renons à l'ohjet principal de cette étude, les variations saisonnicres.

\section{II. - VARIATIONS SAISONNIÈRES}

Pour examiner et caractériser avec précision cet élément des régimes, les débits moyens mensuels applieables à de longues périodes sont le critère fondamental. Mais cela ne suffit point. Il nous faudrait encore les courbes des débits classés de diverses fréquences, partielles ou cumulées, pour chaque mois de chaque année et pour les périodes entières. Car des moyennes identiques, pour des mois différents, peuvent intégrer des variabililés très inégales, et notamment des possibilités très dissemblables d'écarts entre les extrêmes. En général, les Services officiels. pour la plupart des pays, n'établissent point ces rréquences dans les cadres mensuels, ou ils ne publient point les résultats de tels calculs lorsque, par hasard, ils les ont effectués.

Cependant, notre examen aboutirait déjà à des conclusions d'une haute importance si, à défaul des données susdites sur les fréquences, nous possédions des débits moyens mensuels de périodes assez longues pour toutes les principales artères du réseau brésilien. Mais nous ne somnes bien ou moyennement pourvus à cet égard que pour quelques bassins méridionaux et sud-orientaux. Malgré cette insuffisance, qui ne doit en rien faire oublier le très sérieux effort des Services hydrométriques brésiliens et les très beaux résultats déjà oblenus par eux, nous puurons mettre en évidence certains fails essenticls, et pour d'autres, soulever, utilement peut-être, des problemes et poser des jalons.

\section{A) Régime tropical austral}

\section{Généralités.}

Nous commencerons par examiner les types les plus simples, les plus répandus de heancoup, ol marqués par une seule époque de hautes eaux en saison la plus chaude, uno seule phase do basses eaux durant les mois plus frais, une seule hosse, un seul creux dans les graphiques des moyennes mensuelles. Ces types apparliennent pour la plupart au régime tropical austral qui correspond à une distribulion pluviale dont quelques exemples (1914-38) sonl donncs dans le lableau I.

Par le fait qu'ils s'appliquent à trop peu de postes, ces jeux de chilfres donnent d'ailleurs une idee incomplete (et nous reviendrons la-dessus plus loin) des nuances que comporte ce climat aussi bien pour la durée do la saison très pluvieuse, et pour la date du maximum des précipitations que pour la dale du minimum et pour la gravité de ce dernier. Sans trop de simplisme, 
TABIEAU I

\begin{tabular}{|c|c|c|c|c|c|c|c|c|c|c|}
\hline & 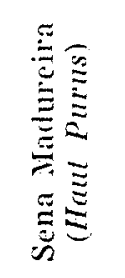 & 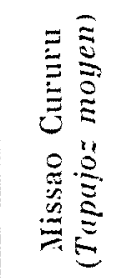 & 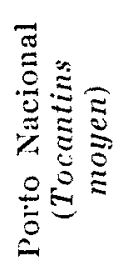 & 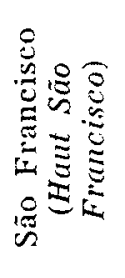 & 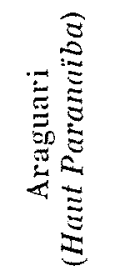 & 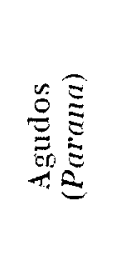 & 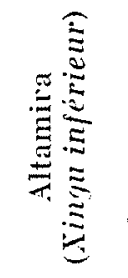 & 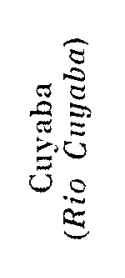 & 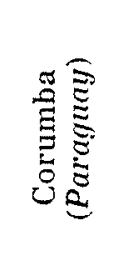 & 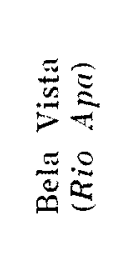 \\
\hline Janvier. & 315,8 & 400 & $29 \cdot 1,2$ & 209,1 & 295,4 & 187,4 & 252,2 & 210 & 183,3 & 160,4 \\
\hline Fevrier. & 301.3 & $4,37,9$ & 297,3 & 168,2 & $2(\mathbf{6} 1,1$ & 148,8 & 272 & 183 & 134,6 & 125,1 \\
\hline Mars. & 257,6 & 418,5 & 386,1 & 151,8 & 227,8 & 99,2 & 303,4 & 223,6 & 115,4 & 112,9 \\
\hline Avril. & 225,1 & 311,4 & 154,5 & 68,8 & 101,7 & 45,4 & $25(j, 9$ & 104,6 & 83,1 & 118 \\
\hline Mai. . & 111,6 & 97.7 & 41,2 & 20 & 16,7 & $4 \cdot 1,2$ & 158 & 43,1 & 65,5 & 121 \\
\hline Juin. . . & 62,9 & 38,7 & 0,4 & 5,7 & 10,7 & 53,4 & 97,6 & 13,7 & 35,2 & 72,3 \\
\hline Juillet . & 32,1 & 40 & 3,2 & 2,3 & 11,1 & 295 & 47 & 9,1 & 19,9 & 38,2 \\
\hline Aoùt. . & 42.2 & 46,7 & 10 & 5,8 & 10,1 & 31,1 & $2 \pi .3$ & 22 & 20,5 & 41,1 \\
\hline Septembre. & 101,3 & 127,8 & 35,3 & $1(i, 5)$ & 13,8 & 66,7 & $.10,9$ & 45,6 & 57,6 & $7: 3.4$ \\
\hline octobies. & 195,1 & 199,3 & 14,3 & $9+1,2$ & 160,9 & 76,9 & 52.1 & 128,8 & 97 & 125,7 \\
\hline Novembre. & 107,7 & $322, \overline{5}$ & 242 & 212 & $2.13,10$ & $86 i, 8$ & 58,6 & 155,2 & 121,2 & 148,5 \\
\hline Décembre... & 294,1 & 329,2 & $287,-1$ & 253,4 & 392,5 & 159,4 & 121,6 & 211,5 & 176,6 & 169,9 \\
\hline Annèe. & $2.136,8$ & $2.769,8$ & $1.795,9$ & $1.207,8$ & $1.785,7$ & $1.054,8$ & $1.687,8$ & $1.350,2$ & $1.114,9$ & $1,309,5$ \\
\hline
\end{tabular}

on peut dire ceci : la sćcheresse est d'autant plus complète en hiver et la saison des pluies d'autant plus courte ( 4 mois, contre 7 plus au nord dans la majorité des lieux) qu'on s'éloigne plus de l'Equateur. A des latitudes assez basses, l'hiver bénéficie d'arrosages encore modestes, mais plus tout a fait insignifiants (quelques dizaines de $\mathrm{mm}$, au lieu de quelques mm par mois). Quant au maximum, il tend à se fixer un peu avant le début de l'été, donc en décembre au sud-est dans les zones du São Francisco, du Paraiba do Sul, du Parana, et à devenir plus tardif (jusqu'en mars), vers le nord.

Ces regimes triomphent, non point comme on Ic verra, sur les régions côtières entre Natal el l'embouchure du Doce, laquelle se trouve vers le $20^{\circ}$ degré de latilude, mais sur des millions de $\mathrm{km}^{2}$ a l'intérieur du pays, au sud de l'Amazone moyen et inférieur, et sur les domaines du Paraguay et du Parana supérieurs. Ils parvienment même jusqu'à la cole sud orientale, sur une large facade, entre Rio de Janciro ef Florianopolis, at peu près.

\section{Parä̈ba do Sul.}

Et c'est dans ce dernier secteur que Ie Paraïba do Sul, une des mieux étudices parmi les rivieres brésiliennes, montre ses caractéristiques à la fois typiques et point particulieres au fleuve en question.

Sur le cours supérieur à Cacapava, les moyennes globales de délits sont assez peu différencées de janvier à mars, avec des coefficients mensuels (rapport des débits en question aux modules annuels), de $1,488,1,664,1,595$. La moyenne maximum se situe en février, ce qui correspond à août boréal. On aura remarqué aussitôt qu'un coefficient mensuel de 1,664 indique un maximum bien peu saillant. Songeons que pour d'autres régimes pluviaux tropicaux, le coefficient le plus élevé peut alteindre beaucoup plus, même en dehors de zones où l'abondance pluviale ne dure pas deux ou trois mois ef oì le sol emmagasine peu de reserves. Sur ces rivières on trouve 4,20 en septembre (sur le Sénégal a Bakel). On note encore 3,5 en septembre pour le Niger à Koulikoro, 2,36 sur Ia Sanaga subéquatoriale à Edea, 4,5 pour la Bénoué à Garoun, 2,2 pour le puissant Oubangui à Bangui, 3,5 pour le Nil Bleu a Khartoum, 2,87 pour le Fleuve Rouge, etc.

Un fait important, el qu'on retrouvera dans les bissins limitrophes de lintérieur, est à signaler : c'est la discordance entre les moyennes mensuclles maxima pour les pluies et pour les débits. Les précipitations doivent être les plus denses en décembre, mais l'écoulement atteinl 


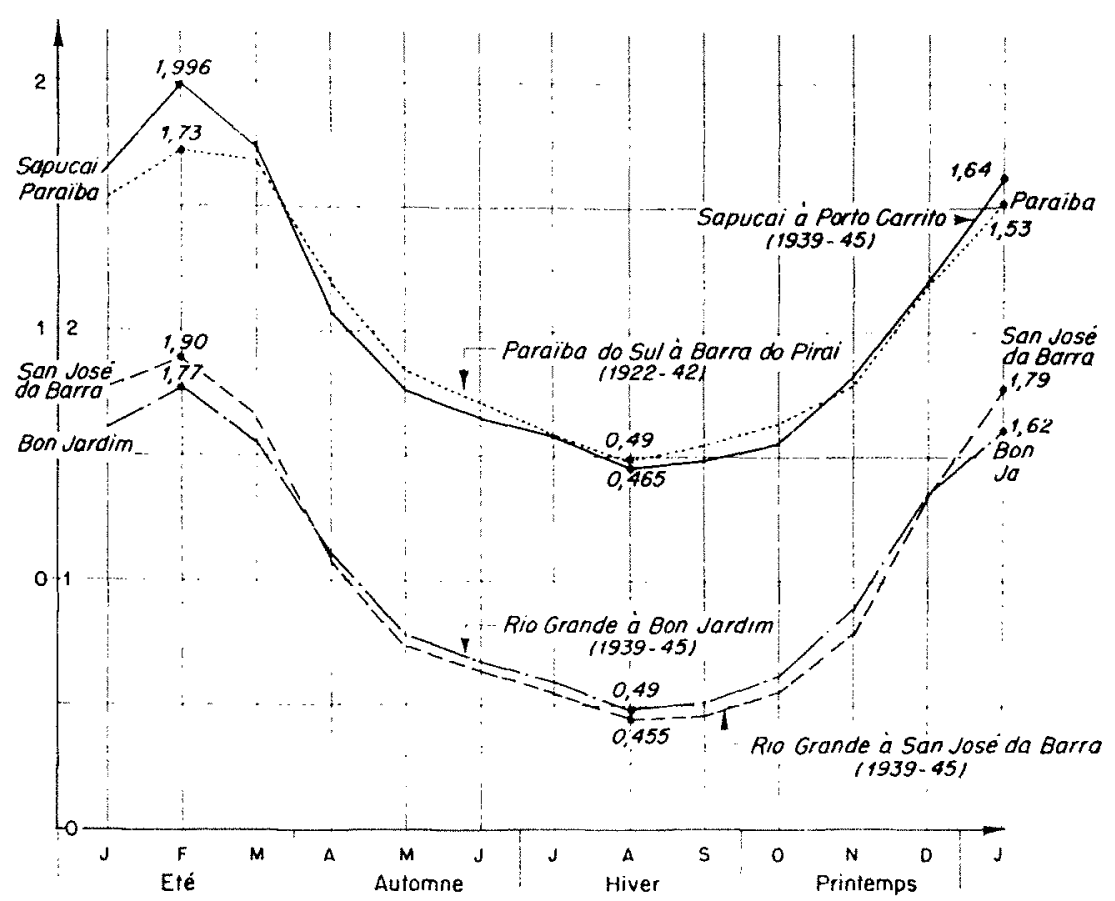

Fu. 3. ... Regime plurial Tropical austral. Coeffeients mensuels de debits.

son apogée deux mois plus tard. C'est qu'en décembre, et malgré l'abondance déjá respectable des chutes d'eau de novembre, les sols, desséchés pendant plusieurs mois auparavant, n'ont pas eu le temps de se saturer, et qu'ils absorbent encore beaucoup d'eau atmosphérique. En janvier, et encore plus en février, sous des précipitations encore très généreuses, l'imprégnation permet un ruissellement bien plus intense et aussi des résurgences abondantes; ce qui revient à dire que le coefficient d'écoulement augmente sensiblement de décembre à février, et peut-être à mars.

D'autre part (et c'est ce qui explique la modeste valeur des coefficients de janvier à mars), la moyenne mensuelle maxima change nettement de mois, suivant les années. En vingt ans, à Cacapava, on la note deux fois en décembre, six fois en janvier, sept en févier, et cinq en mars. En aucun de ces mois on ne peut escompter avec certitude que le débit moyen sera très fort. Je constate 309,7 en janvier 1926 , mais 110,3 seulement, ce qui signifiait de vraies basses eaux en janvier 1936, 415,8 en février 1931, mais 117,5 en février 1936, 402,8 en mars 1923 , et 145,1 en mars 1925. En certaines années, il ne vient pour ainsi dire point de véritables hautes eaux estivales moyennes. Si après la pénurie persistante de janvier-février 1936 on a eu 327,4 en mars, l'été de 1941 n'a point connu de moyennes supérieures à 154,1 en janvier, ni à 156,1 en mars. Bref, point de très hautes eaux régulières, ne manquant pour ainsi dire jamais en un mois donné, et assurant à celui-ci un gros coeffi- eient comme ceux que nous avons énumérés pour les rivières d'autres régions tropicales.

Mèmes caracléristiques estivales à peu pròs, sur le cours moyen à Barra do Piraï en vingl et un ans. Le coefficient mensuel maximum, toujours en février, ne dépasse point 1,73; décembre posside la moyenne maxima trois fois, janvier cinq fois, février sept, et mars six. Les moyennes extrêmes sont 735 et $228 \mathrm{~m}^{3}$ pour janvier, 1.029 et 262 pour février, avec cing valeurs moindres que $400 \mathrm{~m}^{3}$, puis 949 et $306 \mathrm{~m}^{3}$ en mars. L'éte 1941 encore attendit en vain de grosses eaux. Mais ces caprices peuvent ètre qualifiés de régullarité fort louable, en comparaison des vicissitudes ultra-fantasques que nous verrons, an nord-est dans la région eótière vers Bahia el Recife, ou bien au sud-onest, dans le réseau de l'Uruguay ou au voisinage de celui-ci.

En contraste avec la módiocrite relative des moyennes mensuelles maxima, et avec cette variabilité en date et en débit des hautes caux d'une année à l'autre, la saison des basses eaux offre moins d'imprévu. Elle ne comporte pour ainsi dire jamais de crues. Quant à la date des moyennes mensuelles minima, elle est à Barra do Piraï : huit fois août, sept fois septembre, quatre fois octobre, unc fois novembre. Soit en somme un étiage très net dans la denxieme moitié de l'hiver, avec prolongation assez fréquente, quand les pluies tardent, au début du printemps. Sur le cours supérieur à Caçapava, la tendance à la continuation des basses eaux jusqu'à la veille de l'été (décembre 1935, par exemple) est peutêtre plus sensible. Mais seule une étude plus ap- 


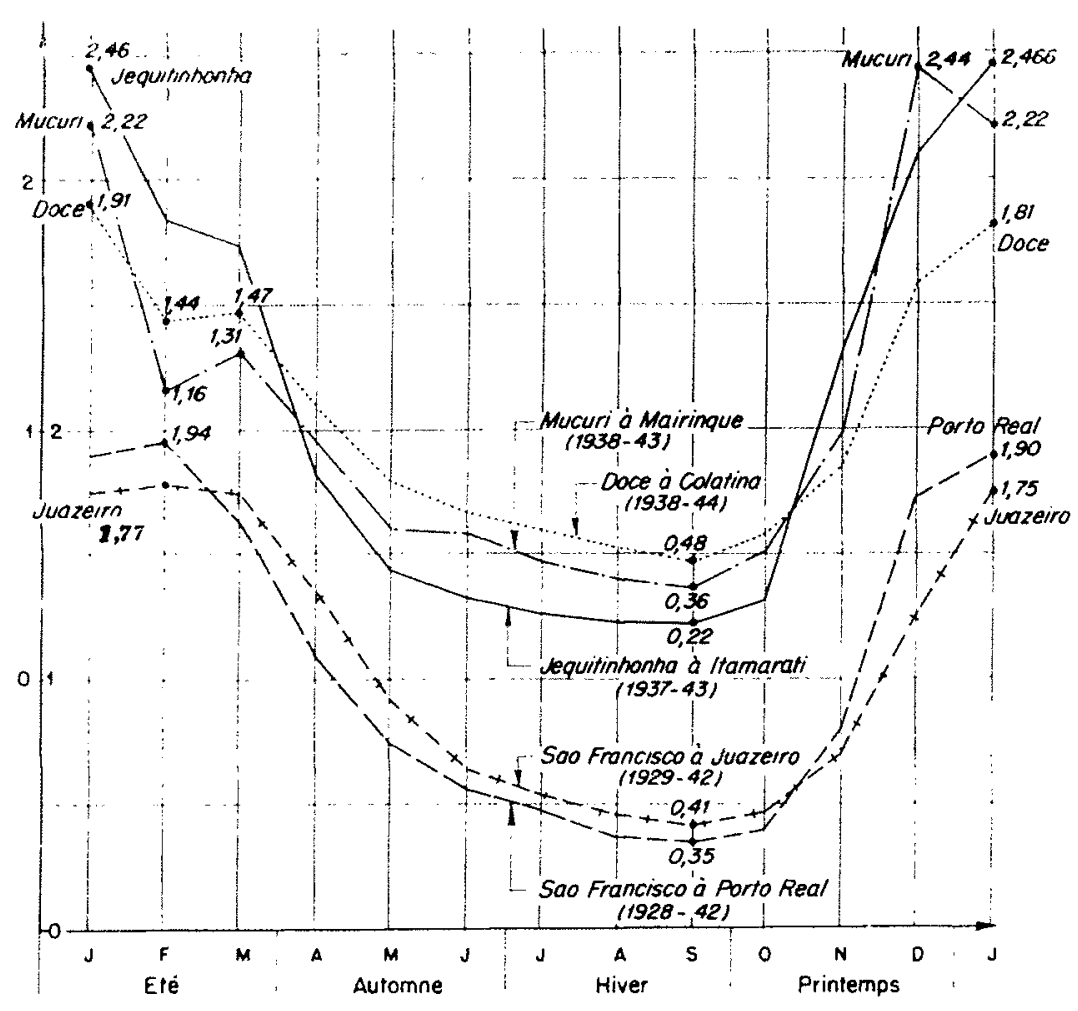

Fig. 4. -- Régime tropical pur (Sato Francisco) ou plus ou moins altéré (Doce, Jequitinhonha, Mucuri). profondie permettrait une conclusion formelle à cet égard.

Dans les pays de régime tropical plus accentué, avec saison pluvieuse plus courte, la moyenne minima au lieu de prendre place dans la deuxième partie de l'hiver, comme ici, a lieu plutôt lors du premier ou même du second mois du printemps, à cause du retard normal des grandes pluies, et du fail que le desséchement antéricur et les températures des mois en question sont plus sévères que dans la région brésilienne iei considérée. D'où plus grosse capacité d'évapotranspiration, aux dépens des premières chutes d'eau bien nourries.

Par exemple, la moyenne mensuelle minima se manifeste en mai (ce qui serait novembre dans l'hémisphère austral) sur le Sénégal à Bakel, en avril sur le Niger à Koulikoro et sur la Bénoué à Garoua, sur la Son, affluent méridional du Gange, et sur le Nil Bleu à Khartoum ou sur son affluent le Sobat.

Cependant la principale caractéristique des basses eaux moyennes dans le bassin du Paraïba n'est pas sa date. C'est l'abondance que conservent les débits moyens mensuels minima; le coefficient mensuel d'août est 0,566 du module à Caçapava, 0,49 à Barra do Piraï. Citons par comparaison : 0,035 sur la Son, 0,10 sur le Nil Bleu, 0,27 sur le Fleuve Rouge à l'origine du della, 0,043 sur le Niger à Koulikoro, bien moins sur le Sénégal.
Si l'on songe à l'infimité des pluies el aux valeurs relativement élevées de la température pendant les quatre à cinq mois hivernaux dans le Brésil tropical, on est surpris d'une aussi bonne tenue fluviale lors de la saison des maigres. Certes, la longue durée et l'abondance des pluies de saison chaude restreignent le temps où les rivières doivent vivre sur leurs réserves soutertaines, et permettent un bon remplissage hydrique du sous-sol. Mais diverses comparaisons montrent que cette explication ne suffit point. On est contraint, comme pour les étiages extrèmes, d'invoquer le pouvoir constitutionnel remarquable de rétention possédé par le sol. Nous retrouverons ce phénomène et son explication, sur de vastes zones voisines.

\section{Rio São Francisco.}

L'allure du régime saisonnier est assez semblable sur le São Francisco, aussi bien à Porto Réal, non très loin de la source qu'à Juazeiro où la surface réceptrice atteint $490.769 \mathrm{~km}^{2}$. Cependant, $\dot{a}$ Porto Real, il y a moins de moyennes maxima en mars ( 1 sur 15) et plus en décembre (4). Le coefficient de janvier égale presque celui de février et décembre l'emporte sur mars. En somme, le centre de gravité, si l'on peut dire ainsi, des hautes eaux est avancé d'à peu près un mois, pour des causes d'ordre pluvial ou géologique. A Juazeiro, les faits relatifs à cette épo- 
que de l'année sont contradictoires. On ne frouve qu'une seule fois sur quatorze une moyenne mensuelle maxima en décembre, mais denx autres chiffres de co mois sont très élevés. Puis une moyeme maxima, la seemde des quatorze ans, apparait en avril contre pas une seule durant ce mois à Porto Real. La variabilité en un mème mois, d'un été à l'autre, est encore énorme. Par exemple je citerai pour mars $7.750 \mathrm{~m}^{3}$ en $1929,2.401$ en 1934. Cette irrégularité empèche les coefficients de débits d'atteindre 2 en moyenne globale pour toute la période. Finalement, c'est encore février qui s'adjuge les plus hautes eaux moyennes, mais avec un très faible avantage sur janvier aux deux stations.

Cependant, sur le cours supérieur, mars sc tient loin au-dessous de férrier $(1,63$ contre 1,94). A Juazeiro, mars égale presque lévrier avec 1,75 , contre 1,777 et 1,752 en janvier. Le fait doit avoir pour cause une accentuation de la pluviosité de mars sur le cours moyen; puis la longueur du cours et le fait que de gros débits passés durant la deuxième quinzaine de février dans le haut bassin ne surviennent que plus tard sur le cours inféricur.

Le centre de gravité des basses eaux est plus tardif que sur le Paraïba. A Porto Réal, 9 moyennes minima sur 15 appartiennent à septembre, et 3 à octobre, 2 seulement à aoùt. A Juazeiro, 10 chiff res de ce genre sont de septembre, 4 d'oclobre, ce qui est le début du printemps. Mais, fait contradictoire et qui mériterait d'ailleurs d'être prouvé avec certitude par des analyses rigoureuses, il semble que la prolongation de réelles basses eaux jusqu'à novembre soit plus rare que sur le Paraïba.

D'autre part, les coefficients de débits des mois à médiocre abondance, quoique plus laibles que sur le Paraiba, demeurent honorables, à Porto Réal $(0,352$ en septenubre) et encore plus ì Juazeiro $(0,406)$ malgré la médiocrité générale de l'alimentation, fait qui tend a affecter le plus les saisons de basses eaux.

Car sous de mêmes répartitions pluviales, en pourcentages mensuels, les coefficients d'ćcoulement de la rivière la moins bien alimentéc tendent à être plus inférieurs à ceux d'autres cours d'eau pour les mois de faibles précipitations que pour ceux d'alimentation généreuse.

Donc là encore les facteurs auxquels nous avons fait allusion plus haut et surtout, semblet-il, l'influence géologique, c'est-à-dire le pouvoir de rétention du sol, contribuent à rendre point trop contrasté, tout au moins pour les moyennes mensuelles, le régime pluvial tropical qui, ailleurs, peut être à tous points de vue excessif.

\section{Bassins du Rio Grande et du haut Parana en GÉNÉRAL.}

Nous retrouvons le même genre de modération, dans le domaine du Rio Grande, qui, nous te rappelons, forme le Parana par sa jonction avec le Pamanaba. Aux stations hydrométriques de ce hassin partiel, les coefficients mensucls globaux les plus fathes que nous ayons caleules ne tombent poinl au-dessous de 0,49 (aoùl) à Bon Jardim (486 km²), de 0,40 (septembre) à Porto Capetinga (25.520 km²), de 0,455 (aoùt) à San José da Barra (52.110 $\left.\mathrm{km}^{2}\right)$; ni de 0,465 (aoùt) à Porto Carrito sur l'affluent le Sapucai. A ces quatre postes les coefficients les plus forts apparaissent en février, malgré la prépondérance des moyennes pluviales de décembre ou au plus tard de janvier, et ils n'atteignent encore nulle part 2 (1,996 sur le Sapucai, 1,77 à Bon Jardim). Janvier vient au second rang partout, sauf sur le Sapucai où mars prend sur lui l'avantage. Tous ees chiffres pour le bassin du Rio Grande s'appliquent par malheur à sept années seulement (1939-45). Les observations d'une bien plus longue période changeraient peut-ètre les classements respectifs de janvier et de mars, puis d'août et de septembre. Mais presque certainement elles laisseraient l'avantage à février, avec des coefficients globaux partoul inférieurs à 2, sauf exceptions insignifiantes. El elles maintiendraient sans doute, pour le mois te plus maigre, des coefficients supérieurs à 0,40 , et plus généralement à 0,45 .

D'après les pluies mensuelles el divers indices hydrométriques (débits et surtout hauteurs d'ean du Parana supérieur brésilien, puis du Parana moyen), nous avons lieu de penser que dans l'ensemble du réseau du Parana, en amont de l'Iguazu, ou tout au moins de l'Ivai, done dans les domaines du Paranaiba au nord-est, puis, sur le Tiète, le Parapanema et leurs branches au sud-est, le régime est presque identique, c'esta-dire pluvial tropical avec maximum d'́té, minimum d'hiver et rapport inferieur at 5 ou 4,5 entre les moyennes mensuelles globales extrêmes. Les plus basses caux ordinaires apparaissent en tout cas en août d'après les moyennes des hauteurs à Puerto Coronel Quito $(618 \mathrm{~km}$ à l'amont de Guayra), et juste à l'aval du confluent du Rio Grande et du Paranaiba, puis à Guayra même. En ce dernier point, lo maximum se fixe sur février avec un faible avantage sur mars, favorisé sans doute par le retard de l'écoulenent et aussi par l'accroissement probable des débits en mars (comme en avril), au sud-est sur l'Ivai et les rivieres voisines (zone de transition avec celles de l'Iguazu el de l'Uruguay où le régime n'est plus du tout pluvial tropical). A Puerto Coronel Quits, le maximum prendrait place en janvier, donc au premier mois de l'été, avec une mince supériorité sur février et sur mars.

Enfin, sur le Paraguay supérieur à Cacères et sur son affluent le Cuyaba, d'apres le livre de M. Soldano, les hauteurs moyennes indiguent un 


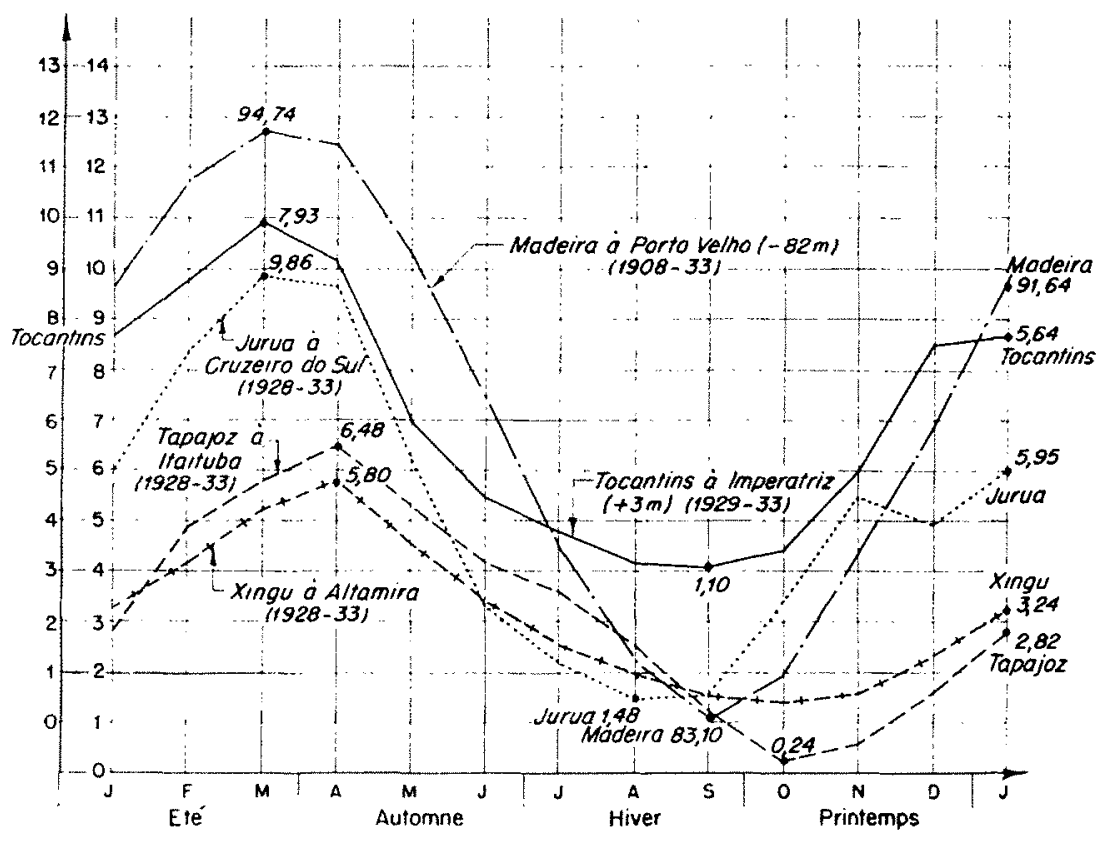

Fig. 5. - Affluents de l'Amazone et rocantins. Régime tropical austral (hauteurs moyennes mensuelles). regime semblable el typique sur lequel nous reviendrons.

\section{BASSIN DE L'AMAZONE.}

Comme nous l'avons déploré, e'est pour le réseau amazonien que nous possédons le moins de données hydrométriques, puisque celles-ci se limitent à des hauteurs aux échelles en un très petit nombre de points.

\section{a) Régime tropical austral typique ou peu retardé.}

Mais ces documents suffisent à nous montrer bien des choses eaptivantes.

Tout d'abord, certaines rivières manifestent un régime tropical austral presque pur, avec moyenne mensuelle maxima fixee en mars. Il en est ainsi pour le Jurua supérieur à Cruzeiro do Sul, et pour le Tocantins, fleuve à part, faut-il dire, à Imperatriz, puis pour la Madeira moyenne a Porto Velho, ef sans doute aussi à Humaita un peu plus en aval, d'après le nombre des maxima mensuels absolus ( 9 en mars, 8 en avril) Le Tapajoz moyen à Cururu aurait même un régime tropical austral encore plus net, grâce à un maximum de février équivalent à août; car je relève en ce lieu, en dix ans, 5 plus hautes cotes annuelles en ce mois, 2,5 en mars.

Dans ces régimes, comme de juste, le minimum moyen appartient à l'hiver, surtout à la fin de cette saison. Il doit survenir en septembre sur le Tapajoz moyen et sur la Madeira, dès août sur le Jurua à Cruzeiro do Sul.

\section{b) Régime tropical it maximum netlement retardé.}

Plus répandu dans l'Amazonic, sur les cours inférieurs des grandes rivières tout au moins, est le type tropical retardé avec maximum d'avril équivalant à notre mois d'octobre. C'est le cas d'après les moyennes mensuelles comme d'après les maxima annuels les plus fréquents, pour le moyen Purus à Labréa (10,5 maxima en avril, 4 en mars, 1,5 en mai), puis pour le Tapajoz inférieur à Itaituba (moyenne de $6,48 \mathrm{~m}$ en avril contre 5,82 cn mars), pour le Xingu inférieur à Allamira (12 maxima en avril, $t$ en mars, 1 en lovrier, 1 en mai), et pour l'Araguiat supérieur (tributaire du Tocantins) à Goiaz $(6,5$ maxima en avril, 2,5 en mars).

Les minima annuels dans ce groupe se manilestent encore en seplembre et octobre, ainsi qu'on pouvait le conjecturer d'après l'étude précédente sommaire que nous avons consacrée aux étiages.

Le retard approximatif d'un mois pour les maxima comme pour les minima (avec moins de nelteté pour ceux-ci), dans le sous-groupe ici considéré, par rapport au précédent, doit tenir d'abord au fait que, saul pour l'Araguia, il s'agit de stations situées plus sur les cours inférieurs que dans les exemples précédents. Le décalage a au moins partiellement pour cause la grande durée de la translation des ondes, chose due à la faiblesse des pentes et à la longueur des cours, et aux tracés du réseau, donc aux caractéristiques du relief, plus qu'à des facteurs climatiques. 
Pour la mème raison, le maximum de la Volga tarde jusqu'au début de juin à Stalingrad, alors qu'il prend place en mai à Kouybichef et à Gorkii; en arril vers les sources du fleuve et sur l'Oka.

Cependant, le maximum moyen d'avril, et done, nous le répétons, du début de l'automne, peut ne pas avoir une origine uniquement morphologique, et s'expliquer aussi par des causes pluviales. Dans la région considéree, et, semblel-il, d'autant plus qu'on descend plus vers le nord (mais les preuves sûres nous manquent faute de postes dans presque toute cette région), les précipitations d'avril, sans égaler celles de mars, sont encore très puissantes et elles bénéficient d'un sol saturé par les surabondantes chutes d'eau des trois mois précédents. Aussi leur écoulement ne doit-il subir qu'un déficit assez médiocre, peut-être le plus faible de n'importe quel mois dans des parties étendues de l'Amazonie. Et le ruissellement considérable qui s'ensuit ajoute ses effets à ceux de la Ienteur sur des distances démesurées pour reporter en avril des maxima qui, dans les cours supérieurs les plus nombreux, auraient lieu plutòt en mars. De la même manière doivent partiellement s'expliquer en Afrique le maximum d'octobre suivi par novembre sur l'Oubangui à Bangui, et celui de novembre dépassant de très peu octobre sur la Sanga à Ơnesso.

\section{B) Régime tropical boréal altéré}

En raisonnant trop vite d'après leur seule situation par rapport au cours amazonien, on pourrait croire que les principaux affluents septentrionaux : Putumayo, Yapura, Rio Negro et Trombetas manifestent un contraste éclatant avec les rivières dont nous venons de montrer les régimes saisonniers du type tropical austral. Les tributaires de gauche offriraient done des régimes tropicaux boréaux, avec maxima franes durant un des mois de notre été, c'est-à-dire en juillet, août ou septembre. La réalité diffère sensiblement de cette vue théorique, pour la raison essentielle que la partie de l'Amazonie située dans l'hémisphère boréal ne dépasse guère en latitude le $4^{\circ}$ degré qui n'est même pas la limite de la zone équatoriale. Et le Yapura pour presque tout son domaine, le Rio Negro inférieur, et

\section{Répartition pluviale de type tropical boréai}

\begin{tabular}{|c|c|c|c|c|}
\hline \multicolumn{5}{|c|}{ Boa Vista (Rio Branco) } \\
\hline Janvier & & 28,7 & Juillet . . . & 323,4 \\
\hline Février. & & 33,1 & Août ... & 173,8 \\
\hline Mars . & & 36,2 & Septembre... & 78,4 \\
\hline Avril & & 84,2 & Octobre..... & 64,7 \\
\hline Mai . & & 283,6 & Novembre... & 48,4 \\
\hline Juin. & & 329,2 & Décembre... & 39,4 \\
\hline
\end{tabular}

le Putumayo encore plus, ne drainent que des zones comprises entre l'Equateur et le $3^{\text {" ou le }}$ $4^{\circ}$ degré sud. En ce territoire, d'ailleur's, le maximum de la pluie doit se situer, d'après deux ou trois stations, en mai plutòt qu'en juin. Cependant au poste le plus septentrional de l'Amazonie brésilienne, Boa Vista sur le Rio Branco, affluent du Rio Negro, les plus grosses précipitations se présentent en juin et celles de juillet sont presque aussi forles; un minimum tres marqué se dessine de novembre à mars : traits conformes, dans une assez large mesure, aux règles du régime tropical nord. Mais les pluies d'anoul sont déjà modérées, puis celles de septembre faibles, comple tenu de la chaleur. Bref, même dans cette région qui n'est qu'une étroite lisière, on ne peut sattendre à trouver des maxima fluviaux en août ou en septembre, comme dans l'Inde, en Indochine, au nord de l'A.O.F., en Ethiopie. EI le maximum moyen de juillet pour les cotes, vers l'origine du Rio Negro inférieur à Barcelos, doit être dû en partie au retard de la translation sur le long cours. Il ne l'emporte que de $4 \mathrm{~cm}$ sur le chiffre de juin. D'autre part, les basses eaux ont lieu en automne (novembre et décembre), bien plutôt qu'en hiver, quoique en cette dernière saison les débits soient encore relativement médiocres. Bref, sur le Rio Negro moyen et inférieur, et sans doute encore plus sur le cours supérieur de cette rivière et dans les parties seplentrionales du Rio Branco et du Trombetas, faute de zones réceptrices à latitude élevée, on troure bien le régime tropical boréal, avec maximum de saison chande, minimum de saison froide, si l'on peut dire ainsi, mais non sans déformations ni décalages. El il paraît assez logique de soupconner que le régime s'abâtardit encore plus au point de devenir presque méconnaissable et de ne plus mériter l'étiquetle de tropical borial, plus au sud, sur le Yapura, le Pulumayo et peutêtre déjà sur l'Uaupés ou Rio Negro supérieur. Pour certains de ces cours d'eau nous supposons même, on va le voir, qu'une hydrologie complexe est possible.

\section{C) Régimes subéquatoriaux ou équatoriaux}

\section{a) Rivitires amazoniennes.}

En Afrique, maintes rivieres congolaises, surtout dans l'hémisphère sud, ont un régime que l'on peut appeler tropical subéquatorial, à deux maxima, l'un à la fin du printemps, l'autre au début de l'automne, parce qu'il y a pour les pluies deux saisons de surabondance plus ou moins séparées par deux saisons sèches.

La distinction se fait encore micux sous l'équateur, et certains petits affuents du Congo ou de ses grands tributaires doivent avoir un régime équatorial conforme à la théorie, avec deux apogées pour les moyennes mensuelles. 
TABIALAU II

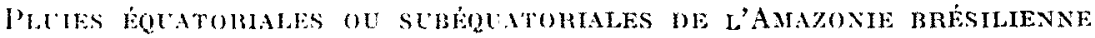

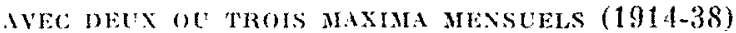

\begin{tabular}{|c|c|c|c|c|c|c|c|}
\hline & 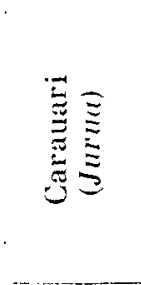 & 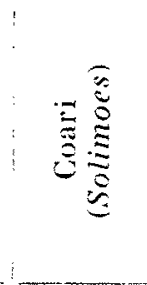 & 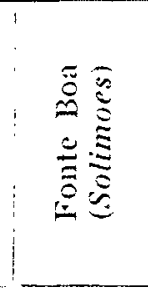 & 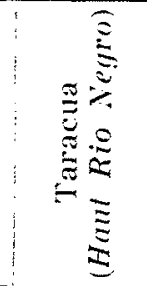 & 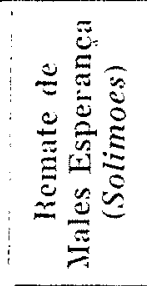 & 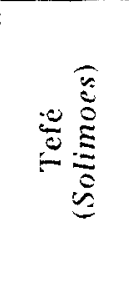 & 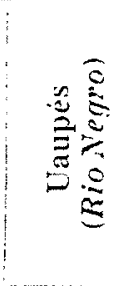 \\
\hline Janvier ....... & $29: 3,6$ & 271,4 & 297,5 & $3.95,5$ & 309,9 & 178.2 & 298,7 \\
\hline Fievrier... & 261,4 & 260,5 & $25 \pi, 1$ & 272 & 281,3 & $25 \%, 2$ & 211,8 \\
\hline Mars... & 326,6 & 299 & 299,3 & 301.9 & $.974,9$ & 249,6 & 281 \\
\hline Arril. & 317,3 & 312,2 & 258,7 & 396,1 & $30-4,1$ & 249,4 & 283,7 \\
\hline Mai... & 211 & 230,9 & 320,5 & 423,7 & 264,2 & 255,6 & 343,6 \\
\hline Juin . . . . & 101,4 & 130,7 & 232,4 & 322 & 158,8 & 168,7 & 238,3 \\
\hline Juillet...... & 62,7 & 64,3 & 186,4 & 317,9 & $12.3,6$ & 98,1 & 229,1 \\
\hline Août. . . . . . & 84,8 & 68,3 & 154,5 & 246 & 134,7 & 87 & 181,8 \\
\hline Sepicmbre..... & 167,6 & 94,7 & 148,1 & 214,1 & $186 i, 6$ & 109 & 169 \\
\hline Octobre... & 225.1 & 156,3 & 169,7 & 198,1 & 236,1 & 149,7 & 164,7 \\
\hline Novembre.. & 206,7 & 159,5 & 220.3 & 232,8 & 234 & 195 & 190,5 \\
\hline Dedectmbre. & 276 & 232,7 & $20.3,9$ & 270,9 & 317,7 & 201,1 & 270,4 \\
\hline Amese & $2.53 .1,8$ & $2.280,5$ & $2.7 .11,4$ & 3.531 & $2.925,9$ & $2.198,6$ & $2.892,($; \\
\hline
\end{tabular}

C'est le cas dans les territoires rançais pour deux affluents du Congo inférieur, la Djoué et la Foulakary, puis par exemple encore, en dehors du bassin congolais pour la Lobé, le Nyong, la Bia.

Existe-t-il des régimes semblables en Amazonie brésilienne? Ici nous devons raisonner d'après le seul indice des pluies, insulfisamment connues. Cependant, plusieurs stations occidentales nous montrent sans équivoque deux ou trois maxima mensuels (tableau II). Mais cela ne semble point ètre la pluviosité équatoriale classique, si netle en des secteurs congolais étendus, à ce qu'il semble, avec deux pointes postéquinoxiales séparées par eing ou six mois, et une relative sécheresse lors de l'un de ces intervalles.

Dans l'ouest de l'Amazonie brésilienne, l'espacement est cerles de guatre mois à Uaupés et à Fonte Boa (janvier et mai), puis plus souvent de deux ou trois mois, à Téfé (Solimoes), à Porto Do Moz, ici par exception vers le bas Xingu, donc très loin de l'Ouest amazonien, et de un mois dans cette dernière zone surtout considérée ici, à savoir à Carauari (Jurua), à Humaita (Madeira moyenne), à Manaos (confluent de l'Amazone et du Rio Nigro), à Remate de Males Esperança, à
São Paulo de Olivença (Solimoes vers les frontières colombiennes ou péruviennes). D'ailleurs à plusieurs postes on note trois apophyses.

Somme toute il paraît s'agir dans cette zone, en ce qui concerne les pluies, d'un simple dédoublement, voire même d'un timide détriplement du maximum d'été austral, avec précipitations encore énormes entre les paroxysmes estivaux trìs rapprochés. Et nous ne jugeons pas impossible que le premier surcroît d'arrosage, bien plus fortement entamé que le second par l'infiltration, n'engendre point un premier maximum distinct sur les rivieres locales.

Le dédoublement des maxima hydrométriques nous paraît, non pas certain (les informations précises et directes nous manquent là-dessus), mais plus possible, sinon probable, d'après le peu qu'on sait sur les pluies et les niveaux hydrometriques, dans la partie du réseau situé entre les confins occidentaux du Brésil, inclusivement, et les Andes. Le fait caractériserait alors les domaines du haut Rio Négro ou Uaupés, du Yapura, du Putumayo, puis plus au sud, le Maranon et l'Ucayali (sans compter des complications ou fausses complications! possibles, causées par des fontes de neiges andines, en ces derniers 
secteurs). Bref, nous ne jugeons pas invraisemblable qu'une hydrologie nettement iquatoriale, avec deux maxima séparés par cing à six mois, existe à l'ouest du réseau amazonien. Elle serait déterminée par des facteurs plus liés à la longitude qu'à la latitude. Mais faute d'observations hydrométriques directes et précises dont nous disposerions, ce ne sont là que des hypothèses, nous n'osons pas même écrire: des faits probables.

\section{b) Fleuves côtiens vers Baha, Recher, Natal.}

Par contre, grâce à des documents précis el assez nombreux fonrnis par de bons Annuares, nous pouvons caractériser en comnaissance de cause les fleuves côliers yui s'échelonnent entre le $18^{\circ}$ et le $12^{\circ}$ degré de latitude à peu pri's, au sud du bas São Francisco.

Les distributions pluviales (Lableau III) dans la zone ici considérée, puis plus au nord jusque vers le $5^{\circ}$ degré, varient d'ailleurs sensiblement sur de courtes distances, d'ouest en est, puisqu'on linit par trouver sur la cote, au sud de Recife, entre le $8^{\circ}$ et le $12^{\circ}$ degié, un maximum pluvial dans la première moitié de la saison froide, en mai-juin-juillet; et en arrière sur des bandes suceessives assez étroites, les plus grandes abondances pluviales se produisent en avril-juin, puis en mars-mai, et ensuite en férrier-avril, ce qui est presque la répartition saisomiere fropicale. En outre, la pluviosilé sur celles de ces zones quasi-méridiennes qui sont les plus voisines de l'Océan, offre plus d'un maximum mensuel: particularité tries importante. Enfin, les bandes plus haul définies, à régimes pluvieux différents, viennent à tour de rôle, en biars, rejoindre l'Ocean au sud de Pahia, puis, mais en moins grand nombre el avee moins de netteti. au nord de Récife: au sud, à partir du $18^{\prime \prime}$ degré, elles sont mème relayees par des bandes nouvelles avec trimestres les plus pluvieux en janvier-mars (pluviosite tout à fait tropicale), puis en décembre-févier, et enfin en novembre-janvier. Cette dernicre zone ćlant très large conlratrie régionalement le retour sur une longue lisiere maritime, du régime pluvial tropical pur, qui rigne (on l'a vu) dans les secteurs de Rio do Janciro, Santos, etc.

Dans la région où les bandes parallèles de pluviosité saisonnières différentes sont les plus ćtroites, se manifestent des régines très variés, sclon le nombre des lanièes traversées par les cours

TABILEAU III

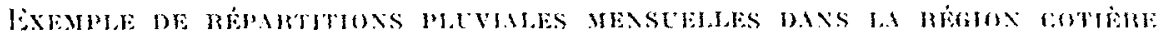

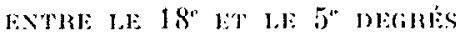

\begin{tabular}{|c|c|c|c|c|c|c|c|}
\hline : & 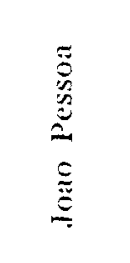 & & 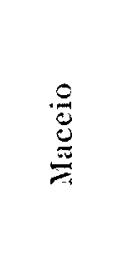 & $\underset{\underbrace{}}{0}$ & 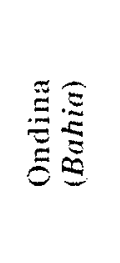 & 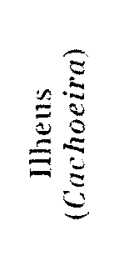 & 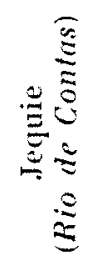 \\
\hline Janvier...... & 72,9 & 25 & 73,1 & 54 & 75,7 & $150, \tilde{)}$ & 45,5 \\
\hline Fevierer...... & 117 & 29,4 & 75,2 & 103,9 & 115 & 170,1 & 88,3 \\
\hline Mars . . . . . . & 180,8 & 15,6 & 137,5 & 134,9 & 168,9 & 249,2 & 118,1 \\
\hline Arril ........ & 258,8 & 95,7 & 183,3 & 223 & 294,6 & 295.9 & 105,1 \\
\hline Mai ........ & 271,1 & 156,3 & 288,8 & 239,5 & 302 & 214.5 & 54,2 \\
\hline Juin . . . . . . . & 311,3 & 153,3 & 301,1 & 278,9 & 220,9 & 154.4 & 12,3 \\
\hline Juillet....... & 198,3 & 153,3 & 218,8 & 174,3 & 192,1 & 180,8 & 51,6 \\
\hline Août........ & 124,3 & 96,4 & 136,7 & 104,7 & 113,2 & 124,7 & 53,2 \\
\hline Seplembre .... & 60,1 & 57,1 & 86,5 & 52,3 & 82,8 & 106,3 & 23,6 \\
\hline Octobre..... & 24,1 & 35,4 & 43,9 & 18,1 & 89,1 & 109 & 38,5 \\
\hline Novembre.... & 28,9 & 34,7 & 27,2 & 23,9 & 111,4 & 189,6 & 111,2 \\
\hline Décembre.... & 45,1 & 42,5 & 44,1 & 29 & 126,4 & 169,7 & 82,5 \\
\hline Anné... & $1.692,7$ & 924,7 & $1.616,2$ & $1.436,8$ & 1.891 .6 & $2.114,7$ & 814 \\
\hline
\end{tabular}




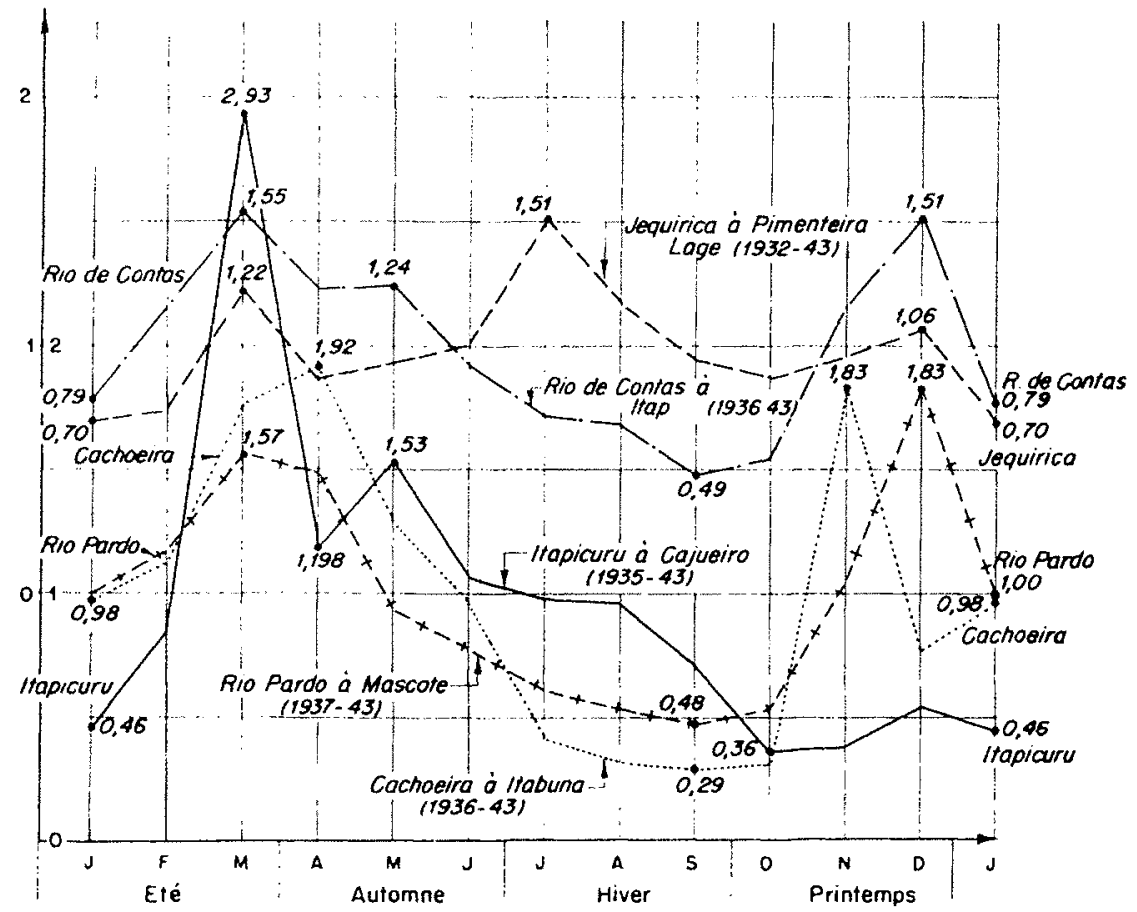

Fig. 6. - Fleuves cotiers de régimes subéquatoriaux variés, au sud de Bahia. avant les stations hydrométriques considérées. Les hydrologies sont d'autant plus contraires aux régimes tropicaux typiques que les sources sont moins enfoncées à l'intérieur et que la diversilé pluviale, en arrière de la cote, est plus marquée.

Commencons done par les rivieres aux regines les plus tropicaux, pour finir par les cas les moins conformes à cette catégorie.

Le Jequitinhonha, qui débouche vers le $16^{\circ}$ degré, a la majeure partie de son bassin évasé dans la zone de pluies tropicales presque classiques. A Itamarati, où il draine $61.907 \mathrm{~km}^{2}$, il n'a qu'un seul maximum, un seul minimum. Et cependant ce fleuve differe assez sensiblement du São Francisco, du Paraïba do Sul, du Rio Grande, du Sapucai par certains détails. L'apogée s'érige en janvier, premier mois estival, et non en février. Puis clle présente un coefficient relativement élevé : 2,457 contre moins de deux pour les maximums mensuels des rivières tropicales plus haul inumérées. Décembre avec 2,097 l'emporte sur mars et même sur février, ce qui est aussi nouveau. Puis les coefficients minima, classiquement situés en hiver $(0,233$ en août, 0,223 en septembre) sont bien plus faibles que sur les organismes précités. Donc hydrologie à maximum plus précoce et moyennes mensuelles plus contrastées que sur lesdits cours d'eau, pour des causes pluviales ou géologiques ou pour ces deux raisons a la fois.

A Colatina, vers le $19^{\circ}$ degré, le Doce dessert un hinterland de $77.407 \mathrm{~km}^{2}$, plus uniforménent arrosés par des pluies de novembre-janvier (donc surtout de printemps) que ce n'est le cas pour le Jequitinhonha, lequel traverse les bandes de pluviosités maxima plus lardives et d'abord netlement tropicales. D'où hydrologic moins classique pour le Doce. De février à mars après le maximum de janvier, il n'y a plus baisse continue mais recrudescence significative, quoique très légère (de 1,44 à 1,475 ) après un affaissement brutal suivant janvier. Comme pour le Jequitinhonha, décembre avec 1,58 offre la plus grosse moyenne apres janvier.

D'autre part, la nature du sol ou une pluviosité mieux répartie atténuent les écarts entre moyennes mensuclles globales extrèmes puisqu'on a 0,477 encore en septembre, contre 1,906 seulement lors du maximum de janvier, valeurs qui rappellent celles du Paraiba do Sul, du Rio Grande, etc.

Puis le Mucuri, situé entre le Jequitinhonha et le Doce, serait presque aussi tropical que lc second, si son bassin, au lieu de manquer d'ampleur (13.820 $\mathrm{km}^{2}$ à Mairinque), empiétait plus dans l'hinterland. Faute de cette condition, si le maximum de décembre $(2,44)$ et les débits encore avantageux de janvier $(2,22)$ sont des traits presque tropicaux, les coefficients de 1,16 pour février et de 1,31 pour mars intriguent par leur médiocrité relative. Mais un fait plus frappant que celle-ci, est qu'ils dessinent une recrudescence, d'ailleurs peu audacieuse, à la fin de l'étí.

Et j’en viens à des régimes bien plus insolites.

Le Rio de Contas, dont l'embouchure se trouve vers le $14^{\circ}$ degré et qui draine à Itapira 52.227 
$\mathrm{km}^{2}$, exhibe son maximum principal (et non plus une recrudescence a peine saillante) en mars $(1,554)$. Son abondance est presque aussi grande en décembre (1,514). Le véritable minimum estival, de 0,792 , et la pénurie plus sensible de la fin de l'hiver et du début du printemps $(0,492$ en septembre), lui composent un remarquable régime complexe à quatre phases.

Le Rio Pardo à Mascote $\left(27.915 \mathrm{~km}^{2}\right)$ a, comme le Rio de Contas, deux maxima et deux minima dans les mêmes mois. Mais grâce à sa situation plus méridionale $\left(15^{\circ}\right.$ au $16^{\circ}$ degré), son indigence d'été n'est point grave; le coefficient le plus faible, celui de janvier, ne s'abaisse point au-dessous de 1. Le maximun principal vient en décembre, comme pour le Mucuri; et avec 1,829 il dépasse nettement la recrudescence de mar's $(1,567$, devant un coefficient presque aussi fort en avil). Le minimum de printemps a presque le mème coefficient que pour le Rio de Contas.

Encore moins conforme que le Contas au type tropical, est l'Itapicuru, qui se termine un peu au nord du $12^{\circ}$ degré et comporte, dans ses $35.123 \mathrm{~km}^{2}$ aboutissant à Cajueiro, des zones appartenant a la région intérieure soumise aux pluies estivales classiques, mais qui draine aussi trop les bandes de pluviosilé changeante pour offrir en définitive un régime tropical. En effet, sil présente un maximum d'ailleurs surfait par la brièveté de la période (coefficient de 2,93) ('n mars $\left(^{\star}\right)$, ses autres coefficients estivaux, par un contraste brutal, sont inférieurs à l'unité : $0,8:)^{4} ;$ en février, 0,46 en janvier, chiffre qui signific pour ce dernier mois une véritable indigence. Puis le débit, retombé violemment au coefficient 1,19 en avil, remonte à 1,528 en mai (**), cœur de l'automne. Enfin, après les basses eaux prinLanières accentuées d'octobre $(0,360)$ et de novembre $(0,397)$, on observe un leger mais curieux exhaussement en décembre $(0,552)$.

Quant à la Cachoiera à Itabuna (vers le $15^{\text {" de- }}$ gré), sa position plus méridionale ne la fait pas plus rentrer que ce n'est le cas pour l'Itapicuru dans le domaine des pluies maxima estivales, car la petitesse de la surface réceptrice $(4.078 \mathrm{~km}-)$ maintient celle-ci entièrement dans la zone côtière. Le régime est donc encore plus différent. du type tropical que ceux du Pardo, du Contas,

(*) Le chiffre exorbitant, pour mars 1940 , de 720,8 millions de mètres cubes (contre 12,037 en mars 1937), contribue beaucoup au rapport de 2,93 . On aurail moins de 2,50 ou méme de 2,25 si les calculs portaient sur vingt ou trente innécs au lieu de neut senlement.

(*) Deja sul le Rio de contas, les coefficients se relèvent de 1,233 en avril a 1,238 en mai. Nous n'avons point signalé plus haut celte recrudescence à peine sensible, et due principalement sans doute, à l'intervention d'une moyenne particulière excessive (celle de mai 1936) dans la courte série de huit annés. Mais nous nosons point affirmer que les observations d'une longue période feraient disparaître à coup sûr cette apophyse légère des moyennes globales. et de l'Itapicuru. En effet, le premier maximum succédant brusquement aux tres basses eaux hiverno-printanières de juillet-octobre $(0,287$ en septembre), surgit en novembre avec 1,828; et aussitot après, en décembre, on observe, au lieu du flot abondant noté sur les rivières précidentes, un embryon de pénurie $(0,767)$. L'aulre maxinum apparaît en avril $(1,92)$ après des caux déjà abondantes de mars $(1,781)$. Pour le coup, moins induement que dans les trois ou quatre régimes précédemment analysés, el malgré la distance appréciable par rapport à l'Equateur, on pourrail qualifier cette hydrologie de pseudo-iquatoriale. Et le graphique des débits moyens mensuels ressemble assez curieusement a celui de certaines rivières congolaises.

Nous arrivons au bout des surprises que peuvent nous réserver ces rivières, dans l'état actuel de notre information, aree le Jequirica, voisin septentrional du Rio de Contas. Un peu plus loin que le $13^{\prime}$ degré, ce bassin, à Pimenleira Lage, ne comprend que $5.000 \mathrm{~km}^{2}$. Or, de 1932 a 1943 , le coefficient mensuel moyen le plus grand appartient a l'hiver 01,52 en juillet). (In second maximum se montre au terme de l'iti (1,225 pour mars) et un Iroisieme peu sensible à la fin du printemps en décombre $(1,058)$, la plus faible moyenne vient an dobul de l'élí $(0,706$ en janvier). Il nous est tres difficile de déterminer dans quelle mesure ces fluctualions suivent celles des pluies, comple tenu des coefficients d'ecoulement et de leurs facteurs thermiques. Il parait assez logique dattribuer le maximum principal de juillet au minimum des temperatures et de l'évapotranspiration, el non ì un paroxysme pluvial qui semble inexistant en ces parages, où les précipitations les plus abondantes fomberaient plutót en mai ou juin.

Nous renoncons en toul cas à chereher un qualificatif pour ce dernier régime, ou a ceux probablement encore plus favorables aux débits de la saison la moins chaude, qui doivent se manifester sur les petits fleuves coliers, plus atu nord, de part et d'autre de Recife, là où le trimestre le plus pluvieux coïncidant presque avec les moins grandes chaleurs est celui de mai a juillel.

Mais nous ne pouvons point en finir avec les rivieres plus méridionales dont nous venons de passer en revue les si diverses et si complexes variations saisonnieres, sans signaler la tres grande irrégularilé que subissent leurs déhits moyens mensuels, d'ume anné à l'autre; fail probablement tres contraire aux principes des climals équatoriatux ou subéquatoriaux atuthenliques. Donc, dans les limites de 8 ans en décembre, le Pardo a debité 74 millions de m* en 1939, 1.071 en 1942. Pour pas plus de 9 mois de novembre, je vois sur le Rio de Contas 16,6 millions de $m^{3}$ en $1939,1.114$ en 1942. Sur 13 mois de décembre pour le .Jequirica, 1940 a connu 7,433 
TABIAEAL IV

\begin{tabular}{|c|c|c|c|c|c|c|}
\hline & 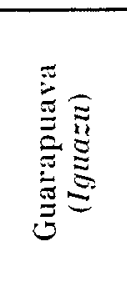 & 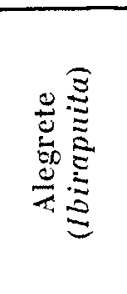 & 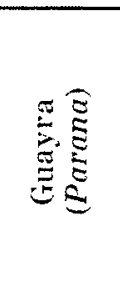 & 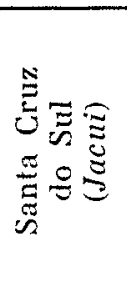 & 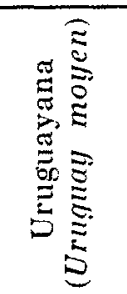 & 芯 \\
\hline Janvier. & 174 & 128,7 & 212 & 144 & 123,5 & 187,6 \\
\hline Fèvier.... & 111,6 & 112,1 & 125,8 & 102,4 & 88,5 & 131,2 \\
\hline Mars...... & 124,2 & 152 & 115,7 & 121,2 & 149,6 & 116,2 \\
\hline Avril... & 114,2 & 152,3 & 129 & 135,1 & $1.12,7$ & 96,1 \\
\hline Mai... & 142,8 & 155,6 & 156,9 & 157 & 123,3 & 115,6 \\
\hline Juin . . . . & 171,3 & 128,4 & 116,9 & 169,1 & 104,3 & 137,9 \\
\hline Inillet. . . . . . . & 88,4 & 106,3 & 64,3 & 145,5 & $G(i, 3$ & 77,6 \\
\hline Août... & 121,9 & 100,1 & 91 & 161,1 & 71,4 & 116,1 \\
\hline Septembre. & 132,8 & 132,9 & 104,8 & 173,8 & 101,9 & 117,4 \\
\hline Octobre... & 172,6 & 138,8 & 157,5 & 128 & 131,9 & 156,6 \\
\hline Novembre. & 199,4 & 117,9 & 144,5 & 110,7 & 91,7 & 122,4 \\
\hline Décembre. & 154,5 & 116,8 & 145,2 & 119,3 & 122,5 & 147,5 \\
\hline Année..... & $1.637,7$ & $1.541,9$ & $1.563,6$ & $1.667,2$ & $1.317,9$ & 1,522 \\
\hline
\end{tabular}

millions de $\mathrm{m}^{3}, 1942,173,510$ millions. Pour la Cachoeira, voici 3,758 millions en novembre 1939 , 419,2 en novembre 1942. Et pour 7 mois d'avril seulement, le Rio Pardo moins fantasque a débité 41,6 millions de $\mathrm{m}^{3}$ en 1939,679 en 1941 . Pour le Jequitinhonha, 430 millions de $\mathrm{m}^{3}$ en décembre 1939 s'opposent à 8.445 millions en 1942. Et nous avons cité des extrêmes phénomémalement contrastés pour l'Itapicuru en mars.

D'une facon générile, il semble que cetle irrigularité diminue dans la mesure où les bassins ont plus d'étendue (facteurs de compensations inferrégionales, généralement) et où les variations saisonnières sont moins éloignées du type tropical classique.

\section{RÉGIMES « SUBTROPICAUX 》 AVEC DEUX SAISONS DE HAUTES EAUX.}

Au sud du Tropique du Capricorne et jusque dans l'Uruguay, au-delà du $32^{\circ}$ degré, on voit triompher d'autres régimes, à deux maxima et deux minima saisonniers, en rapport avec des distributions pluviales compliqućes, très différentes des types tropicaux simples qui règnent, plus au nord et aussi sur la cóle océanique cntre le $23^{\circ}$ et le $28^{\mathrm{e}}$ degré, dans les régions de Rio de Janeiro, São Paulo, et jusque vers Florianopolis.
Ces répartitions diffèrent d'ailleur's plus ou moins de celles que l'on vient de voir produire les résimes à deux ou même trois maxima, de certains fleuves côtiers situés au nord-est de la zone littorale avec maxima pluviaux de pleine saison chaude. Puis elles comportent elles-mêmes toutes sortes de nuances régionales pour les dates des trimestres respectivement les plus arrosés ot les plus secs, et pour divers détails.

Elles s'affirment en maîtresses dans le sud-est de l'empire du Parana moyen [bassin de l'Iguazu et peut-être aussi de l'Ivai (*)] et dans toute l'ctendue des Etats de Santa Catarina et de Rio Grande do Sul (Uruguay et affluents, Jacui, etc.).

Si l'on ne considère que les trimestres les plus riches en précipitations, on voit indiqués pour cette zone, dans une carte de l'Atlas pluviométrique du Brésil : novembre-janvier au nord, puis brusquement, à partir du cours de l'Iguazu ou de ses environs, août-octohre, ou avril-juin, puis juin-août, juillet-septembre, mars-mai, mai-juillet. Il y a là un désordre complet au moins dans les apparences, et en tout cas l'absence d'un dé-

(*) Déjà à Bela Vista sur le Rio Apa, affuent oriental du Paraguay, à la lalitude du Parapanema, done plus au nord que l'Ivai (roir lablenu I), s'esquisse très nettement un second maximum pluvial en automne (avrilmai). 


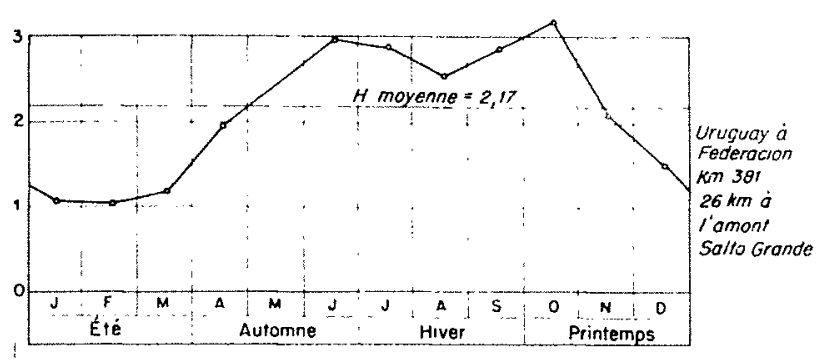

Fig. T. - Hauteurs mensuelles moyennes de l'Lruguay d'après Soldano.

coupage net selon les méridiens ou les paralliles, pour les secteurs successifs. La variété régionale parait encore plus prodigieuse pour les dates du trimestre le moins arrosé. On peut cependant conclure que dans des parties étendues de ces régions, à partir de l'rvai, les pluies tendent à avoir de plus en plus leur maximum plutôt en saison froide et leur minimum plutôt en saison chaude. Quelques exemples locaux montreront cetle variété, si fuyante qu'elle défie les classifications usuelles (Tableau IV).

Pour souligner le caractère étrange, bâtard de ces régimes, on ne saurait mieux faire que de metlre en évidence le fait suivant : à quantité de stations, l'été comporte à la fois, par janvier ou février, un maximum secondaire ou même principal, et un minimum relalif, presque aussilot aprìs, en février ou en mars.

En général l'automne, en mai ou juin, connaîl un ou deux mois très pluvieux. Et on peut faire la même remarque pour le printemps (surtout en octobre). On ne se trompera guere en disant que, selon des modalités de plusieurs sortes, il y a tendance à 3 maxima, en été, en automne, au printemps. Mais les grosses pluies d'été, toul en donnant assez souvent de fortes crues, sont bien plus attaquées par l'évapotranspiration à cause des fortes chaleurs, que les précipitations des saisons plus fraîches. L'hiver connaît en général le minimum le plus accentué pour les pluies. Mais cette faiblesse n'est que relative et ne comporte nulle parl une vraie pénurie, les moyennes génćfales dépassant, partout sans doute, $60 \mathrm{~mm}$ pour le mois le plus sec. Et l'évapotranspiration tombe à ses minima en cette saison, avec des valeurs à vrai dire point petites, car les températures demeurent très lièdes (en juillet, 12 à $14^{\circ}$ sur l'Uruguay, 15 à $16^{\circ}$ sur l'Iguazu, contre 20 à $24^{\circ}$ et 25 à $27^{\circ}$ respectivement en janvier).

On conçoit donc que les régimes tendent a présenter un maximum moyen en automne (mai ou juin), un autre au printemps (octobre dans les cas les plus nombreux, semble(-il). On constate aussi un minimum d'hiver (août ou septembre) et un autre malgré l'abondance pluviale, en été (février ou mars). Par exemple à Alegrete sur l'Ibirapuila, lout à fait at sud-ouest du pays, en 1941-49 actobre a $174 \mathrm{~m}^{3}$, mai 173 , seplembre 91,3 ; mars, pas plus de 19,7. Pour l'Uruguay inféreiur à Federacion, les niveaux d'aoùt sonl triss supéricurs à ceux do févier et ne marquent pas une véribable pénurie, mais sembment une legere reduction de l'abondance entre les deux saisons des plus hautes eaux. L'Iguazu à Tipo, non loin en amont de sa chute grandiose, est presque aussi faible en aont (hiver) qu'en mars. Mais cet cquilibre relatif entre les deux périodes d'indigence ne doil apparaitre qu'au nord, sur celte rivière et sur l'lvai moins sùrement, on sur une bande citroite au sud de l'Iguazu. Les régimes de ces derniers cours d'eau doivent done former la Iransition entre la zone tropicale, où prédominent les débits du semestre le plus chaud, et la zone méridionale, où l'Uruguay et ses tributaires doivent dre dans l'ensemble sensiblement les plus gonflés durant le semestre frais, mai-octobre.

En somme, et cest un fait curieux, on trouve dans toute cette zone méridionale des régimes assez semblables à coux des rivieres nivo-pluviales et plurio-nivales francaises ou ilaliennes seplentrionales, fris sensibles aux pluies médiermancennes d'autome et qui éprouvent aussi des hautes eaux printanieres. Mais le maximum sudbresilien de printemps ne doil pas une goulle d'eau a la fonte des neiges. Il s'asil idei d'umo hydrologie entièrement pluviale, pour lacpuelle un qualificatif n'est encore point facile à trouver. Disons du bout des lives et sans aucune admiration pour le torme que c'est, si l'on reut, un rigime pluvial "sublropical *.

Il ne contraste pas de maniere éclatante avee les types « pseudo équatoriaux $\gg$ que l'on a vus vers Bahia. Mais il s'oppose beaucoup plus radialement a thydrologie tropicale plus ou moins pure dont les inaxima d'été ou du début de l'automne triomphent sur une grande partie du Brésil.

Selon les sous-régions dont on a esquissi la différencialion au point de vue des répartitions pluviales mensuelles et lrimestrielles, et sans doute aussi selon les groupes d'amnées, les classements respectifs des débils moyens mensucls de printemps ou d'aulomne, peut-être même encore les dates do maxima en question doivent

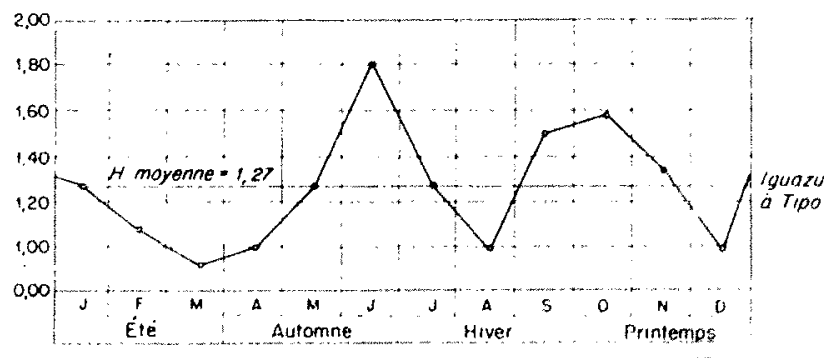

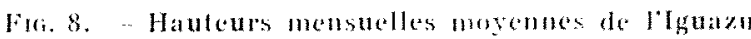
d'apres Soldano. 
pouvoir varier, surtout en ce qui concerne le printemps.

Quant aux ecarls entre haules et basses eaux moyennes globales, ils doivent différer encore plus d'après les régions, et pour plus d'exactitude sans doute, surtout d'apres les pouvoirs d'emmagasinement du sol. Par malheur, pour les débits moyens mensuels de ces rivières, nous sommes mal documentés. On peut cependant signaler que l'Ibirapuita est près de neuf fois plus fort en octobre qu'en mars, chiffre à peu près double de ceux qu'on a soulignés pour maintes rivières du type tropical.

Sur l'Iguazu, d'après des moyennes de trois ans à Porto Uniao (début du cours moyen), le rapport n'atteindrait pas tout à fait 4 , ce qui peut être une exception en cette zone. Mais je n'estime point du tout certain que dans l'ensemble du bassin de l'Uruguay le rapport des moyennes mensuelles globales extrêmes soil excessif, et qu'ainsi ce fleuve contraste de manière flagrante avec la pondération dont témoignent à ce point de vue le Parana et nombre de ses affluents. Cerles, d'après la très grande faiblesse des ètiages et malgré la possibilité de crues en été, on peut supposer sans trop de risque que les moyennes mensuelles globales minima ofrent de pauvres coefficients. Mais nous ne sommes point sûrs que les coefficients globaux les plus avantageux soient élevés, ni mème qu’ils atteignent 1,75. Car les hautes caux, d'après les moyennes, s'étalent sur deux saisons, ou pratiquement (dans le cours inférieur de l'Uruguay faute de creux intermédiaire profond) sur un semestre, et cela ne peut se faire qu'aux dépens du coefficient le plus élevé. Cet écrêtement se réalise grâce à des différences pluviales bien plus capricieuses, pour un même mois, dans le système de l'Uruguay que dans celui de Parana. C'est en cela, dans les valeurs successives des moyennes pour les mêmes mois, à travers plusicurs années, bien plus que pour les moyennes mensuelles globales des différents mois comparéc les unes aux autres, que les rivières étudiées dans ce paragraphe manquent de ponderation. On ne saurait trop redire à ce propos que les climats tempérés ne sont pas du tout ou guère, pour leurs pluies, des climats réguliers, avec répartitions mensuelles semblables à elles-mêmes daus la suite des millésimes. La réalité se montre pour cux généralement toute contraire. Il peut, il doit y avoir bien plus de régularité dans les contrastes pluvieux saisonniers sous des climats tropicaux typiques. Et nous avons vu cela se traduire dans les débits du Rio Grande, du Paraïba, etc.

A cet égard, les inconstances de l'Ibirapuita ne font probablement que refléter, peut-être point arec une exagération très grande, la tendance du réseau uruguayen dans son ensemble. En neul ans, les moyennes particulières des deux mois les plus riches ont varié à Alegrete comme de 1 à 31 en mai, comme de 1 à 14,5 en octobre; celles du mois le plus pauve (mars) ont fluctuc comme de 1 à 7 ; et janvier, mois de débits encore maigres $\left(41 \mathrm{~m}^{3}\right)$, a connu $4,8 \mathrm{~m}^{3}$ en $1945,178 \mathrm{en}$ 1944. Pour quarante ans et cinquante ans et plus, les rapports dépasseraient peut-être 20 ou 30 pour tous les mois et 50 pour plusieurs d'entre eux.

Mais, sous de mêmes conditions climatiques, selon toutes chances, les moyennes mensuelles particulières ou globales les plus riches seraient un peu moins gonflées, les moyennes les plus pauvres supérieures de beaucoup (en pourcenlages), et les rapports des moyennes extrêmes bien moindres (proposition qui résulte des considérations arithmétiques les plus élémentaires), si le sol possédait un pouvoir d'emmagasinement supérieur à la maigre faculté que révèlent, en ceci, les étiages.

\section{D) Régimes complexes changeants des plus grands fleuves}

\section{GÉNÉralités.}

Plus un bassin fluvial est situé à cheval sur des zones climatiques différenciées, plus son résime évoluera de l'amont à l'aval; car les affluents successifs le modifieront plus ou moins sraduellement et sérieusement selon le dessin du réseau, l'échelonnement de leurs confluences, les aires respectivement drainées par eux et par la rivière principale, et les variations saisonnières de celle-ci et d'eux.

C'est l'hydrologie complexe changeante. Elle comporte sans doute, au long des cours, des perlurbations très importantes, sinon des subversions totales par rapport aux régimes des branches supérieures, pour les fleuves côtiers voisins de Bahia. Nous avons noté précédemment quelques résultats de ces vicissitudes, il serait lrop long ici (et peut-ètre difficile) d'en suive les phases cerlainement captivantes.

Pour les tres grandes rivières brésiliennes, ces transformations doivent en principe n'être point Irès spectaculaires. Car le Parana, comme l'Amazone, ont beau dérclopper leur réseau sous des climats point identiques pour la totalité de chacun des bassins en question, les surfaces où prévaut le régime tropical austral pur ou déformé l'emportent de manière écrasante sur les aires où se développent d'autres influences. Et cette relative similitude rend secondaires les retouches produites dans les régimes, au fil de l'eau, par les affluents hétérogènes.

\section{Parana.}

Tout d'abord, l'hydrologie tropicale simple a maximum estival et basses eaux d'hiver, l'emporte presque sans lutte ni nuances appréciables 
sur 650.000 à $675.000 \mathrm{~km}^{2}$ du Parana brésilien. alors qu'au confluent avec l'Iguazu, ce flewve draine à peu près $770.000 \mathrm{~km}=$.

Et si l'Ivai et le Pequeri ont une tendance au régime subtropical à 2 maxima, chose point démontrée, ce phénomène à Puerto Guayra ne se manifeste point ou guère, sauf peut-ètre par une décroissance encore plus lente qu'à l'amont, des hauteurs moyennes (et par conséquent des débits) entre mai et juin. $200 \mathrm{~km}$ plus loin, les hauteurs à Puerto Bertoni traduisent sans équivoque l'inlluence perturbatrice de l'Iguazu. Le miximum automnal de cette rivière remplace par un palier, sur le fleuve, la décroissance peu accentuéc mais encore nette que nous venons de noter de mai i juin pour Guayra. Fait plus significalif, plus apparent sur le graphique, octobre, date de l'autre maximum de l'Iguazu, comporte une recrudescence du Parana en ce mois, avant une courle baisse en novembre. Ainsi l'Iguazu, hien qu'il ajoute seulement $1.200 \mathrm{~m}^{3}$ au module sept à huit fois plus fort du Parana, donne à celui-ci un régime à deux maxima, dont l'un n'est qu'une très médiocre apophyse, à vrai dire. Et les hautes eaux de l'été tropical austral, el la médiocrité hivernale si frappantes aux stations d'avan! l'Iguazu, demeurent de beaucoup les traits prédominants. En somme, l'hydrologie tris simple du Parana s'est très légèrement détraquée avant même que le Paraguay n'intervienne de maniére déconcertante (et d'ailleurs avec des effets point décisifs). Mais le cas du Paraguay est une autre histoire que nous reprendrons plus loin.

\section{Amazone.}

Alors que le régime saisomnier du Parana se complique seulement à l'approche du cours inférieur, celui du formidable Amazone se montre, non sans quelque supercherie, le plus simple vers l'aval, que nous considérons en examinant, d'abord, les cotes à Obidos.

Rien de plus enfantin apparemment. En six ans (1928-33), le maximum vient en mai, dont la cote moyenne dépasse de très peu $(6,36 \mathrm{~m}$ contre $6,30 \mathrm{~m}$ ) celle de juin. Le minimum se place en novembre $(0,86 \mathrm{~m})$. Les indications fournies par les hauteurs extrêmes de chaque mois de chaque année en vingt ans (1928-47) confirment celle indication : 11 maxima apparticnnent is mai, 9 ì juin. La régularité pour les dates est moindre en ce qui concerne les ćtiages. Sur 18 chiffres hien nets, 8,5 reviennent à novembre, 4 à octobre, 3,5 à décembre, 2 à janvier, 1 à septembre (mais c'est le niveau le plus faible de tous). L'interprétation est assez facile; d'abord pour les hautes eaux. Le maximum de mai-juin, ou plus exactement sans doute du 10 mai an 10 juin, c'est avant tout, survenant avec un grand retard

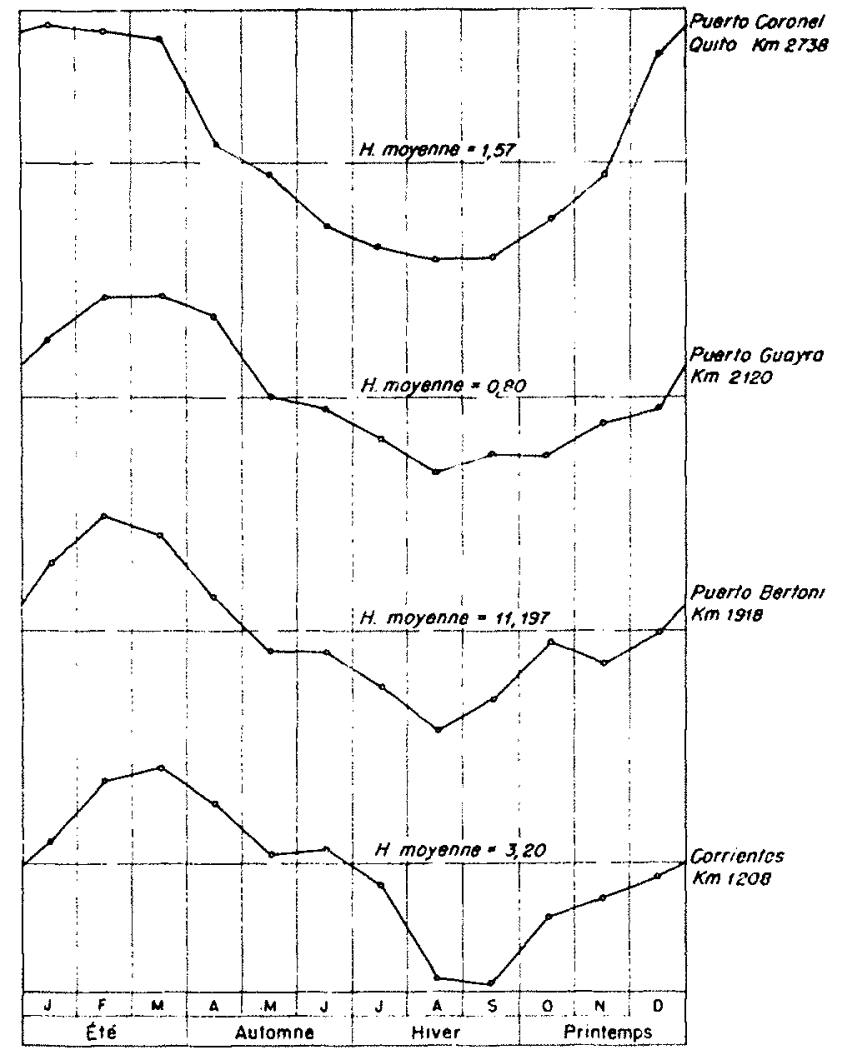

Fia. 9. -.- Mauleuts moyemes mensuelles du parama diupres Soldano.

sur les ruissellements locatux maxima, par l'efrel des longues distances el de la déclivité, puis des inondations ennemies des translations rapides, le flot tropical austral noté en avril (done deja avec un décalage vers l'aulomne) sur le Jurua et le Purus moyen, ef en mars sur la Madeira movenne. Le regime tropical boreal, point parfaii, du Rio Négro et l'hydrologie sans doute plus hybride, mais point tris dimerente, du Yajuara, n'ont pu contrecarer autrement que par des retouches lemprise de la lendance austrike. Car d'abord, les bassins de ces rivieres, el surfoul leurs parties situeses an nord de l'Eyuateur, seml bien moins vastes que fes contrées méridionales de l'Amazonie. Puis, sur le Rio Nesgro inférieur, ru la répartition particulière des pluies, les débits d'avril sont deja imposants. Les écoulements probablement encore plus riches de mai, sur to Trombetas, puis sur les affluents occidentaux de rive gauche, et selon les donnces hydrometriques directes, sur le Rio Negro, aident l'Amazne d'Obidos, compte tenu des temps de transtalion, a rester presque aussi abondante en juin quien mai. Et le maximum tropical boréal de juillet ou de la fin de juin, dans la partie la plus septentrionale du bassin, ne fait que ralentir la baisse des eaux amazoniennes en juillet et en aont.

Par contre, plus Ioin qu'Obidos, vers l'ocian, 


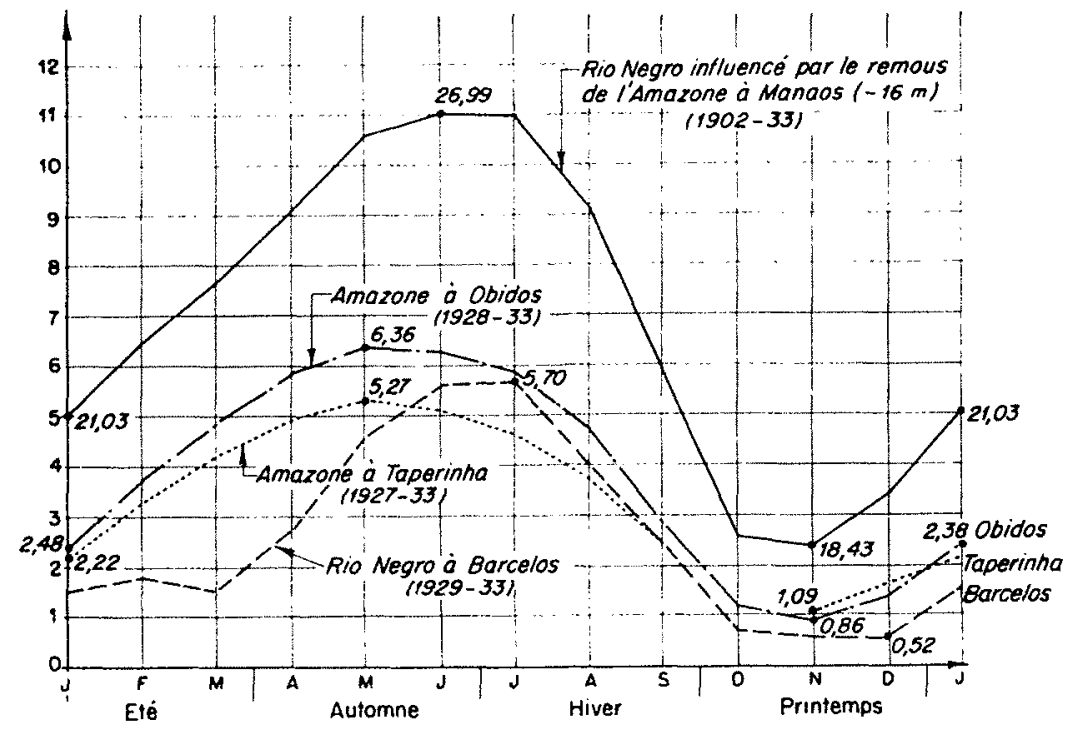

Fic. 10. - Régime lropical nord et Amazone inférieur. Hauteurs moyennes mensuelles. a Taperinha ou Santarem, juste après le confluent avec le Tapajoz, l'avantage de mai sur juin passe de 6 a $15 \mathrm{~cm}$. Donc il se révèle, si la conformation de la courbe hauteur's-débits ne dément point l'impression créée par les cotes, plus sensible qu'à Obidos. Et l'infériorité d'avril sur mai se réduit de 49 à $27 \mathrm{~cm}$. L'ívolution serait inverse, avec juin égalant mai, sans doute, si le seul facteur de transformation était le déplacement peu rapide de l'onde vers l'aval. Done un facteur nouveau est intervenu pour créer lat légère modification exposée. C'est certainement le fait que le Tapajoz à Itaituba présente son maximum, cependant retardé lui-même par la distance, comme on l'a vu, en avril, ef qu'il baisse ensuite très vivement (de $1,09 \mathrm{~m}$ entre avril et mai, puis de 1,20 entre mai et juin). La contraction de son renfort combal la tendance opposée qui se manifeste sur le Rio Négro, et peut-être aussi sur le Yapura et le Trombetas.

Pas plus de mystère pour la saison des basses caux. Le fait essentiel est non seulement que le rígime tropical austral est prépondérant pour elles comme pour les gros débits; mais encore que, pas plus que pour ces derniers, il n'y a contraste absolu pour les étiages, avec décalage voisin de six mois, entre le régime des rivières septentrionales et celui des affluents méridionaux. Les sommets et les creux des graphiques pour les uns et pour les autres ne sont séparés que par deux à trois mois, en gros. En effet, comme nous l'avons déjà vu, les rivières septentrionales, et qui le sont d'ailleurs bien peu, n'ont pas leur minimum en plein hiver et au début du printemps (pour elles ce serait de novembre is avril ou mai). Leur affaiblissement se prononce surtout en automne boréal (d'octobre à décembre); ainsi, ce minimum avancé coïncide, à peu d'intervalle près, avec celui des rivières tropicales australes, plus classique quoique lui-même retardé, puisque les creux annuels boréaux, en Amazonie, prennent place d'aoùt à décembre, ou de seplembre à janvier. C'est celte analogie partielle des régimes respectifs, opérant de chaque côté de l'Amazone, qui donne à celle-ei, après le conlluent avec la Madeira, une apparence aussi simple.

Mais il ne faut pas s'y fier. Examinons, ou tâchons de supputer ce qui se passe avant le confluent avec la Madeira, et d'abord sur un court troncon entre l'embouchure de celle rivière et celle du Rio Négro. A Manaos, sur celui-ci, mais à proximité de l'Amazone qui cxerce un remous, le maximum de juin, avec un avantage de $2 \mathrm{~cm}$ seulement sur juillet, mais de $36 \mathrm{~cm}$ sur avril, exprime peut-être, non seulement le régime du Rio Négro, mais encore, par compromis, celui du fleuve principal. Entre le Rio Négro et la Madeira, done, l'influence boréale introduirait un avantage modique, mais significatif de juin sur mai, au lieu du contraire à Obidos et à Taperinha. Mais le semestre des basses eaux doit être presque le mème à Manaos qu'aux deux stations d'aval. A l'amont le plus proche, entre le confluent du Putumayo et celui du Rio Négro, exclusivement, il se peut qu'on retrouve presque en tous points l'hydrologie essentiellement tropicale sud retardée, qui prédomine en aval de la Madeira. Car selon de fortes chances, le puissant Yapura doit différer encore moins du Purus el du Jurua faute de situation vraiment septentrionale pour lui, que ne s'opposent par exemple la Madeira et le Rio Négro. Mais plus à l'ouest encore, il n'est pas impossible, comme on l'a vu, que se manifeste sur les branches péruviennes et colombiennes du réseau une hydrologie équa- 
toriale à deux maxima et que le facteur neige (*) opérant sur les Andes ajoute un élément de complexité foncière. Dans ce cas, le Maranon et même sa continuation brésilienne, le Solimoes, jusqu'aux confluents avec le Putumayo ou le Jurua, auraient peut-être, comme le prétendent certains auteurs, deux périodes de hautes eaux séparées par deux phases d'indigence bénigne.

D’après DEnis (Géographie Universelle, A. Colin), le Maranon péruvien aurait une poussée de décembre à février, une autre d'avril à juillet. Et même à Téfé, plus en aval, après les confluents du Jurua et du Yapura, le Solimoes roulerait, ses hautes eaux en octobre-janvier, puis de mars à juillet. Nous aimerions savoir si ces assertions se fondent sur des chiffres hydrométriques sérieux ou sur des constatations faites à l'ceil. On voit quels éléments d'ailleurs incertains de com-

(*) Si ce facteur agit, c'est surtout sur le haut Maranon, enserré et entoncé dans les Andes sur plus de $600 \mathrm{~km}$ de distance, puis sur le tiers supérieur de l'Ucayali, et à un degré bien moindre dans l'extrến'couest pour de petites parties des bassins du Napo, du Putumayo, du Yapura. Les lempératures clevées et uniformes, ou peu différenciés, sous ces latitudes, doivent restreindre à très peu de chose ou abolir l'alternance de Ia rẻtention, $p$ uis de la fonte. De toutes facons, les pulsations fluviales dues à la neige sont limitées à de irop petites surfaces pour jouer un rôle marquant à côté des résultals des lluctuations saisonnières pluviales sur le Solimoes et déjà, peut-être, sur le Maranon inferieur.

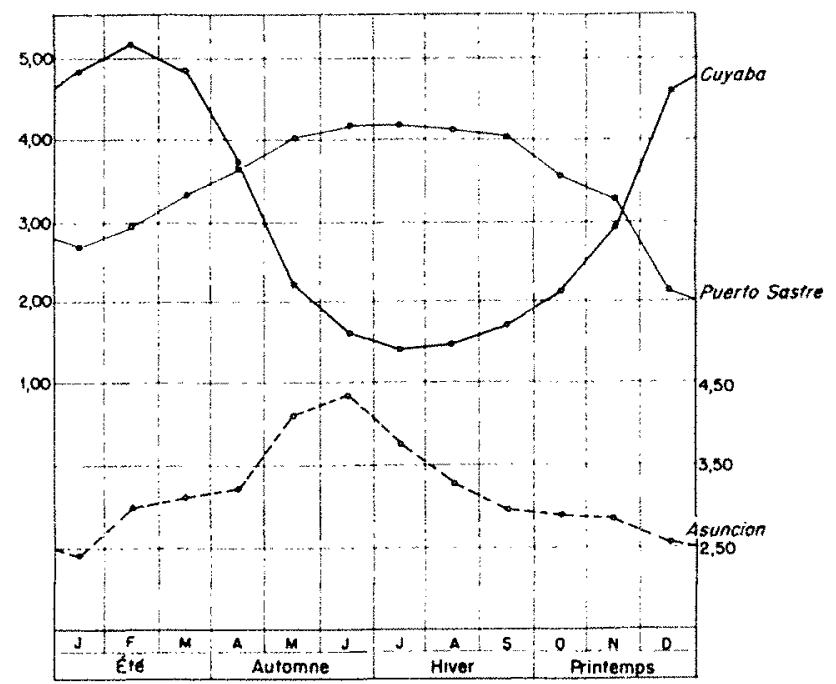

Fia. 11. - Hauteurs moyenties mensuelles du Paraguay d'après Soldano.

plexilé peuvent agir dans la moilié, ou plus sûrement dans le tiers occidental du bassin el comment la simplicile d'Obidos el de Taperinha peut etre géographiquement, of jusqu'à un certain degré, un masque.

Il imporle de fare ici ressortir qu'aucun trompel'oil de ce genre ne fonctionne sur le bas-Congo, second fleuve mondial et que l'on compare souvent à l'Amazone, parce que l'Equateur traverse

TABLAL B. - BASSIN DJ: L AMAZONE. - HAUTEURS MOJENTES MENSTEIAES

\begin{tabular}{|c|c|c|c|c|c|c|c|c|c|c|c|c|}
\hline & Janv. & Févr. & Mars & Avril & Mai & Juin & Juil. & Aoút & Sept. & Oel. & Nov. & Déc. \\
\hline $\begin{array}{l}\text { Jurua à Cruzeiro do Sul........ } \\
\qquad(1928-1933)\end{array}$ & 5,95 & 8,32 & 9,86 & 9,64 & 6,35 & 3,35 & 2,21 & 1,48 & 1,60 & 3,34 & 5,43 & 4,95 \\
\hline Rio Negro à Barcelos (1929-1933). & 1,53 & 1,80 & 1,55 & 2,84 & 4,51 & 5,66 & 5,70 & 4,02 & 2,44 & 0,68 & 0,58 & 0,52 \\
\hline Rio Negro à Manaos (1902-1933). & 21,03 & 22,46 & 23,63 & 25,18 & 26,63 & 26.99 & 26.97 & 25,15 & 21,79 & 18,55 & 18.43 & 19,38 \\
\hline $\begin{array}{r}\text { Madeira à Porto Velho........... } \\
\qquad(1908-1933)\end{array}$ & 91,64 & 93,70 & 94.74 & 94,45 & 92,29 & 89,50 & 86,69 & 84,30 & 83,10 & 83,98 & 36,40 & 82,75 \\
\hline Tapajoz à Itaituba (1928-1933). & 2,82 & 4,80 & 5,82 & 6,48 & 5.39 & 4,19 & 3,53 & 2,53 & 1,13 & 0.24 & 0.53 & 1.56 \\
\hline Amazone à Obidos (1928-1933). & 2,38 & 3,75 & 4,89 & 5,87 & 6,36 & 6,30 & 5,82 & 4,78 & 2,82 & 1,06 & 0,86 & 1,35 \\
\hline Xingu à Altamira (1928-1933). & 3,24 & 4,17 & 5,24 & 5,80 & 4,57 & 3,47 & 2,50 & 1,98 & 1,58 & 1,42 & 1,58 & 2,36 \\
\hline Amazone à Taperinha (1927-1933). & 2,22 & 3,29 & 4,23 & 5,00 & 5,27 & 5,12 & 4,69 & 3,86 & 2,45 & 1,22 & 1,09 & 1,54 \\
\hline $\begin{array}{l}\text { Tocantins à Imperatriz......... } \\
(1929-1933)\end{array}$ & 5,64 & 6.73 & 7,93 & 7,24 & 3,99 & 2,41 & 1,68 & 1.26 & 1.10 & 1,42 & 2,91 & 5,49 \\
\hline
\end{tabular}


les bassins géants de l'un et de l'autre. Lui aussi éprouve surtout l'influence des pluies tropicales ou subéquatoriales d'hémisphère sud. Mais celles-ci ne se répartissent pas du tout comme en Amérique. Elles connaissent, jusqu'à proximité de l'Océan, en général deux maxima traduits par les variations des rivières, comme le Congo Supérieur et le Kassaï, avec prédominance du flot d'avril-mai (automne) sur celui d'octobre-décembre (printemps), pour le premier ct le contraire pour le second. Fn outre la Sanga, et surtoul l'Óubangui, venus plus franchement que le Rio Négro de l'hémisphère nord, ont un maximum tropical boréal non classique (ce qui le placerait en juillet-août ou septembre) mais relardé sur octobre (tandis que celui du Rio Négro est avancé sur juin). En définitive, la surabondance septentrionale unique d'octobre-décembre se joint avec une faible avance au second flot des rivières australes. D’où prédominance très sensible pour le Congo inférieur du maximum de décembre sur celui de mai (ondes retardées par les distances). Or e'est presque exactement en octobre-décembre que l'Amazone inférieur connaît son unique phase de basses eaux. Et en mai, tandis qu'il culmine, le bas Congo, tout en éprouvant son premier gonflement distinct, reste bien plus faible qu'en décembre (à Léopoldville : 2,96 m contre $4,85 \mathrm{~m}$ ). Mais à Stanleyville, il roule plus d'eau en avril-mai qu'en novembre-décembre. Presque sur tout son cours, les observations hydrométriques, infiniment plus nombreuses que dans le bassin de l'Amazone, y compris force jaugeages, révèlent deux saisons de hautes eaux, deux époques de faiblesse avec des différences notables d'amont en aval entre les classements respectifs, les ordres de succession des quatre moyennes menstuelles respectivement les plus fortes et les plus faibles. Or nous avons yu que sur la majeure partie du cours de l'Amazone, au Brésil lout au moins, le graphique des moyennes mensuelles ne présente qu'une seule bosse et un seul creux.

\section{Paraguay.}

Si l'on voulait préjuger des facteurs d'après les seules apparences hydrométriques, les transformations du régime paraguayen entre l'amont et l'aval nous abuseraient terriblement.

Le point crucial est qu'au rebours de ce qu'on observe tout au long du Parana et de l'Amazone inférieur, l'allure des variations saisonnières change sur le Paraguay du tout au tout. En effet, dans le haut bassin, à Cuyaba sur le Rio de ce nom, et à São Luis de Caceres un peu plus au sud, sur le Paraguay lui-même mais avant le confluent avec le Cuyaba, l'hydrologie est, comme on l'a déjà dit, tropicale typique avec maximum de février, minimum de juillet-août. Puis à Corumba, soit $740 \mathrm{~km}$ au sud de São Luis de Cace. res, on observe presque l'inverse avec maximum de juin, minimum de décembre-janvier. A Puerto Sastre, peu après la sortie du Brésil, le maximum encore retardé et surtout aplati s'étale de juin à août avec un très léger avantage pour juillel, le creux est en janvier. Or, aucun changement sensible des régimes saisonniers dans les affluents n'a pu produire cette interversion sensationnelle. I a cause n'ofre cependant aucun mystère. C'est l'étalement des eaux estivales, chaque année, sur le champ d'inondation du « Pantanal », long de $1.200 \mathrm{~km}$, et submersible, de par sa conformation, sur une largeur moyenne de plusieurs dizaines de kilomètres. D'où, la très faible déclivité de 3 à $4 \mathrm{~cm}$ par $\mathrm{km}$ aidant, le décalage de quatre à cinq mois, par rapport aux stations du bassin supérieur, dans le graphique des moyennes hydrométriques mensuelles. Sur le cours inférieur, hors du Brésil, quelques affluents de régime tropical apporteront leur flot principal en été. Ils créeront d'abord une poussce en février, suivie d'un palier de février à mars, loin au-dessous du maximum retardé par la morphologie du talweg. Plus loin, après la jonction avec le Pilcomayo et le Bermejo venus des Andes, l'apport de ces rivières aura créé de hautes eaux en plateforme, de février à juin.

\section{CONCLUSION}

Nous nous bornerons pour conclure à exprimer le vœu que les observations hydrométriques modernes, et notamment les bonnes mesures de débits, soient étendues à l'ensemble du réseau lluvial brésilien y compris le systeme de l'Amazone. Certes, les jaugeages sur des rivieres aussi abondantes, larges et profondes, et en particulier sur le gigantesque fleure principal, présenteront des difficultes graves, mais probablement toutes surmontables dans l'état actuel de la technique, très coûteuses à vrai dire. Et l'évidence de cet inconvénient pratique, rendu plus lourd par l'infimité du peuplement et par la léthargie industrielle et commerciale de l'Amazonie, peut détourner les Administrations intéressées de l'efrort à consentir. Nous avons le ferme espoir que, dans un avenir proche, ces objections seront balayées par une curiosité scientifique dont les fruits, tôt ou tard, et comme toujours, profiteront à l'économie nationale brésilienne. 